\title{
A Systematic Review and Appraisal of Epidemiological Studies on Household Fuel Use and Its Health Effects Using Demographic and Health Surveys
}

\author{
Daniel B. Odo ${ }^{1,2, * \mathbb{C}}$, Ian A. Yang ${ }^{3,4}$ and Luke D. Knibbs ${ }^{1}$ \\ 1 School of Public Health, The University of Queensland, Herston, QLD 4006, Australia; 1.knibbs@uq.edu.au \\ 2 College of Health Sciences, Arsi University, Oromia, Asella P.O. Box 193, Ethiopia \\ 3 Thoracic Program, The Prince Charles Hospital, Metro North Hospital and Health Service, Chermside, \\ QLD 4032, Australia; i.yang@uq.edu.au \\ 4 UQ Thoracic Research Centre, Faculty of Medicine, The University of Queensland, \\ Brisbane QLD 4032, Australia \\ * Correspondence: d.bogale@uq.edu.au; Tel.: +61-424306479
}

check for updates

Citation: Odo, D.B.; Yang, I.A.; Knibbs, L.D. A Systematic Review and Appraisal of Epidemiological Studies on Household Fuel Use and Its Health Effects Using Demographic and Health Surveys. Int. J. Environ. Res. Public Health 2021, 18, 1411. https://doi.org/10.3390/ ijerph18041411

Academic Editors: Judy S. LaKind and Carol Burns

Received: 19 December 2020

Accepted: 28 January 2021

Published: 3 February 2021

Publisher's Note: MDPI stays neutral with regard to jurisdictional claims in published maps and institutional affiliations.

Copyright: (c) 2021 by the authors. Licensee MDPI, Basel, Switzerland. This article is an open access article distributed under the terms and conditions of the Creative Commons Attribution (CC BY) license (https:// creativecommons.org/licenses/by/ $4.0 /)$.

\begin{abstract}
The domestic combustion of polluting fuels is associated with an estimated 3 million premature deaths each year and contributes to climate change. In many low- and middle-income countries (LMICs), valid and representative estimates of people exposed to household air pollution (HAP) are scarce. The Demographic and Health Survey (DHS) is an important and consistent source of data on household fuel use for cooking and has facilitated studies of health effects. However, the body of research based on DHS data has not been systematically identified, nor its strengths and limitations critically assessed as a whole. We aimed to systematically review epidemiological studies using DHS data that considered cooking fuel type as the main exposure, including the assessment of the extent and key drivers of bias. Following PRISMA guidelines, we searched PubMed, Web of Science, Scopus and the DHS publication portal. We assessed the quality and risk of bias (RoB) of studies using a novel tool. Of 2748 records remaining after removing duplicates, 63 were read in full. A total of 45 out of 63 studies were included in our review, spanning 11 different health outcomes and representing 50 unique analyses. In total, 41 of 45 (91\%) studies analysed health outcomes in children $<5$ years of age, including respiratory infections $(n=17)$, death (all-cause) $(n=14)$, low birthweight $(n=5)$, stunting and anaemia $(n=5)$. Inconsistencies were observed between studies in how cooking fuels were classified into relatively high- and low-polluting. Overall, 36/50 (80\%) studies reported statistically significant adverse associations between polluting fuels and health outcomes. In total, 18/50 (36\%) of the analyses were scored as having moderate RoB, while 16/50 $(32 \%)$ analyses were scored as having serious or critical RoB. Although HAP exposure assessment is not the main focus of the DHS, it is the main, often only, source of information in many LMICs. An appreciable proportion of studies using it to analyse the association between cooking fuel use and health have potential for high RoB, mostly related to confounder control, exposure assessment and misclassification, and outcome ascertainment. Based on our findings, we provide some suggestions for ways in which revising the information collected by the DHS could make it even more amenable to studies of household fuel use and health, and reduce the RoB, without being onerous to collect and analyse.
\end{abstract}

Keywords: cooking fuel; household air pollution; health effects; Demographic and Health Survey; DHS; low- and middle-income countries

\section{Introduction}

The World Health Organization (WHO), in its indoor air quality guidelines, defines solid fuels, including coal and biomass (e.g., charcoal, wood, dung and crop residues), and kerosene as "polluting". Households that use these fuels are exposed to household 
air pollution (HAP) [1]. Despite progress towards universal access to clean household energy, $47 \%$ of global households ( 3.6 billion people) continue to depend on polluting fuels [2]. Combustion of these fuels in the household, for cooking, heating and lighting, emits particulate and gaseous pollutants that harm human health, contribute to increased ambient air pollution, and affect climate change [1,3]. It is estimated that women and children incur the greatest health burden, due to spending more time in and around the home in low- and middle-income countries (LMICs) [4,5].

Exposure to air pollution is the fourth-leading risk factor for disease burden worldwide, accounting for nearly 7 million premature deaths and more than 213 million disabilityadjusted life-years (DALYs) in 2019 [6]. Each year, an estimated 3 million premature deaths and 91 million healthy years of life are lost due to illness attributable to HAP alone [6]. These HAP-related deaths and DALYs are nearly two times higher in countries with low socio-economic status [7]. HAP from cooking with polluting fuels is associated with many adverse health outcomes, of which the highest level of evidence is for cardiovascular and respiratory diseases, diabetes, cataracts, low birthweight and short gestation (preterm birth) $[1,6,8]$.

Broad-scale evidence on the health burden of exposure to HAP in LMICs comes mainly from national surveys because the ideal methods, such as biomarkers, personal and micro-environmental monitoring are not feasible in those settings. The Demographic and Health Survey (DHS), which is funded primarily by the United States Agency for International Development (USAID), is a nationally representative household survey that has been used to collect data on population, health and nutrition in more than 90 LMICs since 1984 [9]. It is the main, often only, source of essential data in LMICs. DHS data have been used to calculate more than 30 indicators, supporting tracking the Sustainable Development Goals (SDGs) [10,11]. DHS data are crucial in a wide variety of research activities and policy decisions, including the allocation of health resources [12].

Globally, estimates of the proportion of the population using polluting fuels [1], and the resultant health risks in LMICs, is based on data collected primarily by DHS surveys $[6,13]$. In those countries, exposure to HAP is assessed indirectly using the type of fuel used for cooking as a proxy [14]. Numerous studies have reported associations between HAP and adverse health effects by performing secondary analyses of DHS data. The DHS surveys are periodically revised by soliciting feedback from end users with content expertise. Several extensive reviews and meta-analyses of HAP and health effects included DHS-based studies along with other relevant non-DHS studies [15-19]. However, to our knowledge, no reviews have focused solely on DHS-based epidemiological studies of HAP.

Here, we aimed to: (i) identify and collate all relevant peer-reviewed epidemiological studies of DHS-derived HAP estimates and health performed globally, (ii) determine what variables and analytical approaches can place studies at greater risk of bias, using a novel rating tool, and (iii) identify additional variables that could boost utility without being onerous to collect in resource-limited settings, given that the main purpose is not to collect data for HAP research. Due to practical constraints, this review did not include articles published in languages other than English.

\section{Materials and Methods}

\subsection{Literature Search}

The systematic review followed the approach detailed in the Preferred Reporting Items for Systematic Reviews and Meta-Analyses (PRISMA) guidelines [20]. Three electronic databases, including PubMed, Web of Science, and Scopus were used to search peerreviewed articles. The DHS program publication portal and the reference lists of the included primary studies and review articles were searched for additional studies.

Medical Subject Headings (MeSH), free-text terms and keywords used in the search include air pollution (household air pollution, indoor air pollution and indoor), survey characteristics (family characteristics, household characteristics, health surveys and cross- 
sectional studies) and cooking activities and fuel types (cooking, indoor cooking, outdoor cooking, kitchen, fuel, cooking fuel, heating fuel, wood, kerosene, charcoal, coal, biomass and solid fuel). These were combined with Boolean operators "AND/OR" and the search strategy was customised to each database (Table S1). The search strategy was developed in consultation with a librarian experienced in systematic reviews of epidemiological studies. The initial search was undertaken in June 2019, and an updated search, restricted to articles published after that date, was performed in September 2020. The updated search was done for PubMed only.

\subsection{Review Protocol and Inclusion Criteria}

The detailed methods of the analysis and inclusion criteria were pre-specified and registered in the PROSPERO international prospective register of systematic reviews (registration number: CRD42019137937) [21]. In brief, peer-reviewed studies that were based entirely on DHS data and considered HAP as a main exposure variable were included, with no restriction on the type of health outcome, geographic location (country), age or gender of study participants. All searches were restricted to articles published in English since the inception of the DHS program (1985) through to September 2020.

The search results from the main databases and the DHS publication portal were imported into EndNote X9. Duplicate records were then removed. The first reviewer (D.B.O.) screened the title and abstract of the remaining records against the inclusion criteria. A second reviewer (L.D.K.) independently reviewed titles and abstracts of a randomly selected subset comprising $10 \%$ of the overall records (i.e., after removal of duplicates). If disagreement occurred, it was resolved by further discussion to reach a consensus regarding inclusion or exclusion. In the course of data abstraction, authors of two articles were consulted for detailed clarification.

\subsection{Risk of Bias and Quality Assessment}

The quality and risk of bias (RoB) of studies meeting the inclusion criteria were assessed, using a new RoB instrument for non-randomized studies (NRS) of exposures, [22] by one of the authors (D.B.O.). This tool seeks to assess the methodological quality of the evidence and the RoB of studies of environmental exposures. It compares the quality and RoB of each study with a hypothetical randomised target experiment, rather than a study-design directed quality and RoB assessment approach [22]. The three steps involved in applying this instrument were: (1) present the review question, potential confounders, and exposure and outcome measurement accuracy information, (2) describe each eligible study as a hypothetical target experiment and including specific confounders from that study that will require consideration, and (3) assess RoB across seven items on the strengths and limitations of studies. Detailed guidance on the application of the tool is available elsewhere [22], including how to interpret and present the RoB of the studies, and using that information to make a transparent judgment.

The seven domains of RoB items used include bias due to confounding, bias in the selection of participants, bias in the classification of exposure, bias due to the departure from intended exposure, bias due to missing data, bias in the measurement of outcome, and bias in the selection of reported results. The final judgment for each RoB item was rated as low, moderate, serious or critical [22] (Table S2). As a general indicator, a "low" RoB study would control important confounders listed in the RoB assessment table, would ascertain health outcome objectively (with validated method) and supported with record linkage (Table S2). Such studies that scored higher RoB, particularly studies that were labelled under critical RoB were expected to be dropped. However, because our aim was to identify and collate all DHS-based studies on household fuels and health, including the benefits and weaknesses of using DHS data, we did not exclude any studies based on RoB scores. We instead used them to highlight some of the important considerations and challenges of using DHS data and various analytical methods used by the different studies. 
We conducted the RoB assessment at the outcome level. Because RoB can vary within a study if multiple outcomes are assessed. We, therefore, evaluated RoB for each outcome.

\section{Results}

\subsection{Search Results and Screening}

The PRISMA flow chart is shown in Figure 1. The main database search returned 3396 records, plus 21 studies obtained from the DHS publication search portal. Of the 2748 records left after the removal of duplicates, 63 full-text records were downloaded for further assessment. The remaining 2685 records were excluded because they were not relevant to our review (see Figure 1 for details). Of the full-text records reviewed, 45 (71\%) studies met our inclusion criteria (Figure 1). Tables 1-6 contain a detailed summary of the included studies, including year, country, health outcomes, statistical methods, sample size, among others.

\subsection{Description of Included Studies}

Overall, data from 57 countries were included in these 45 studies. Three of 45 studies assessed multiple health outcomes [23-25], while the remainder assessed a single outcome, for a total of 50 unique analyses. All studies were cross-sectional in design. The country most frequently analysed was India (13/45 studies) [25-37], followed by Nepal (4 studies) [38-41], then Bangladesh [23,24,42], Nigeria [43-45] and Pakistan [46-48] (3 studies each). South Africa $[49,50]$ and Zimbabwe [51,52] each accounted for two studies, respectively. The remaining studies focused on Ghana [53], the Philippines [54], Tanzania [55], Swaziland [56], Uganda [57], Afghanistan [58] and Malawi [59].

For multi-country analyses, a study of child mortality included 47 countries [60]. Three studies [61-63] combined multiple data from different sub-Saharan African countries and the other three studies pooled data from countries in different regions [64-66].

The included studies were published between 1999 and 2020, with the majority covering the period 2013-2020. The DHS data used by these studies were collected between 1992 and 2018. ARI in children is the most frequently reported health outcome (17 studies) followed by the studies of under-five mortality (including neonatal, post-neonatal, infant and child) (14 studies). Figure 2 presents a summary of the included studies by the number of publications, types of health outcomes and year of publication (Figure 2).

\subsection{Quality and Risk of Bias Assessment Results}

Based on the study-level RoB assessment, no study scored low RoB. Eighteen out of $50(36 \%)$ analyses had moderate RoB, 16/50 (32\%) analyses had serious RoB, and the remaining 16/50 (32\%) analyses had critical RoB. The main contributors to the relatively high proportion of studies with serious and critical RoB at the study-level were inadequate control of known or potential confounders, methods of exposure assessment and outcome ascertainment, and misclassification of exposure. Based on the item-level RoB assessment, three of the seven domains, where all the studies included in this review scored low RoB, were on the selection of participants, missing data and selection of reported results, whilst confounding and the classification of exposure (i.e., separating households into those using relatively clean and relatively polluting fuels) were the two domains that resulted in serious or critical RoB. The results of RoB assessment and grading for each study and item-level are presented in the Supplementary Files (Table S3). 


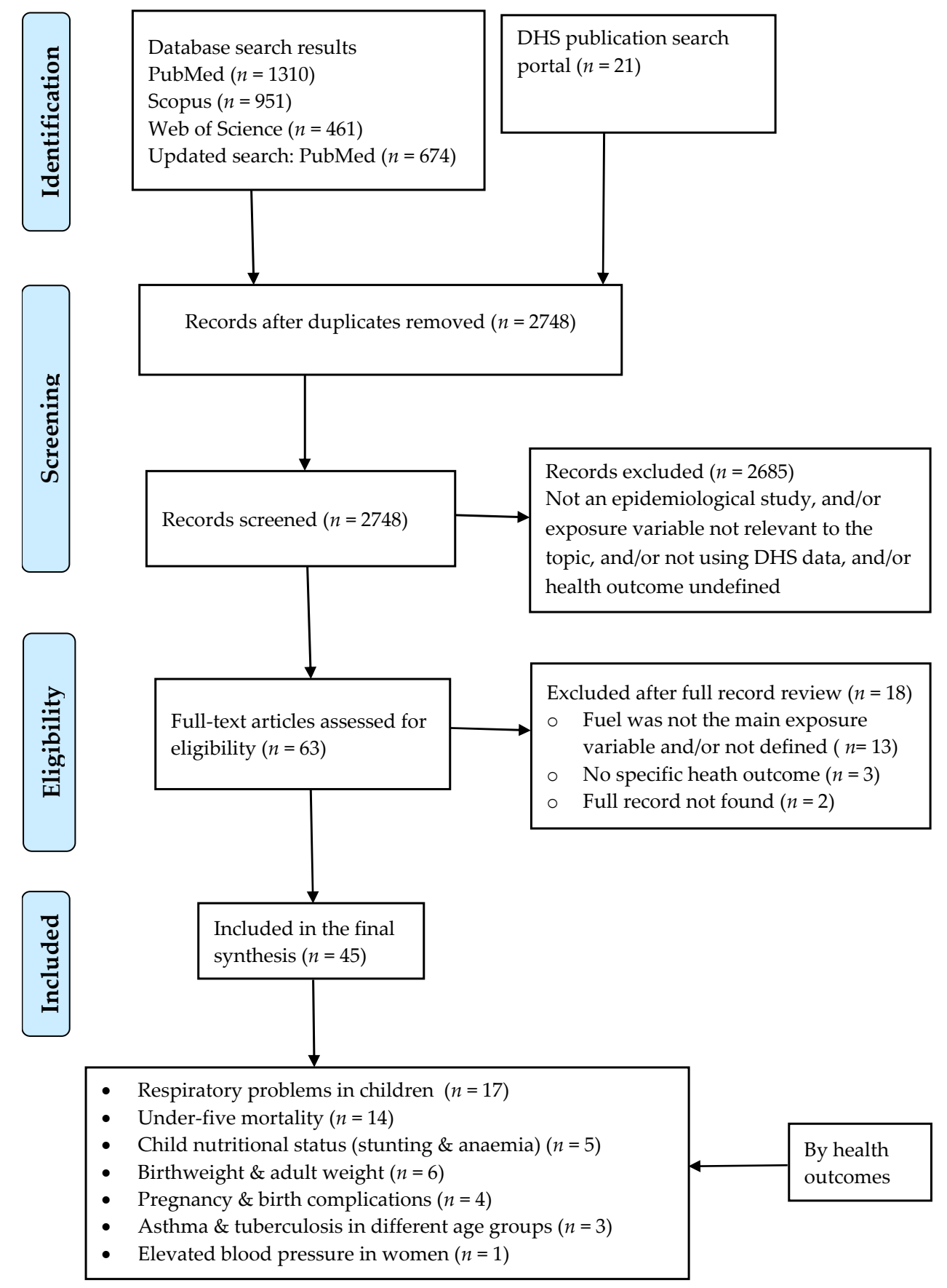

Figure 1. The flow of studies from identification to data extraction from databases based on the PRISMA guidelines. Note: three articles reported seven health outcomes, together. 
Table 1. DHS based evidence on the association between cooking fuel use and acute respiratory infection in children.

\begin{tabular}{|c|c|c|c|c|c|c|c|c|}
\hline $\begin{array}{c}\text { Authors and } \\
\text { Publication Year }\end{array}$ & $\begin{array}{l}\text { Country and } \\
\text { Survey Year }\end{array}$ & Sample Size & Exposed Group & $\begin{array}{l}\text { Comparator } \\
\text { Group }\end{array}$ & $\begin{array}{l}\text { Outcome: Case } \\
\text { Definition }\end{array}$ & $\begin{array}{c}\text { Covariate } \\
\text { Adjusted (See } \\
\text { Footnote) }\end{array}$ & $\begin{array}{c}\text { Statistical } \\
\text { Method and } \\
\text { Main Results }\end{array}$ & $\begin{array}{c}\text { Risk of Bias } \\
\text { Score }\end{array}$ \\
\hline $\begin{array}{l}\text { Mondal et al. } \\
\text { (2020) }\end{array}$ & $\begin{array}{c}\text { India } \\
\text { 2015-16 }\end{array}$ & 247,743 & $\begin{array}{c}\text { Biomass fuels: } \\
\text { kerosene, } \\
\text { coal/lignite, } \\
\text { charcoal, wood, } \\
\text { crop waste, } \\
\text { straw / shrubs/grass, } \\
\text { animal dung and } \\
\text { others }\end{array}$ & $\begin{array}{c}\text { Clean fuels: } \\
\text { electricity, LPG, } \\
\text { natural gas, and } \\
\text { biogas }\end{array}$ & $\begin{array}{l}\text { ARI in children aged } \\
<5 \text { year: cough } \\
\text { accompanied by short, } \\
\text { rapid, or difficult } \\
\text { breathing that is chest } \\
\text { related }\end{array}$ & $\begin{array}{c}1,2,3,14,15,30 \\
31,45,47,49,52 \\
\text { and } 78 a\end{array}$ & $\begin{array}{c}\text { Logistic regression } \\
\text { OR, 95\% CI: } \\
1.10(1.01-1.20)\end{array}$ & Moderate \\
\hline Naz et al. (2020) & $\begin{array}{c}\text { Pakistan } \\
3 \text { waves } \\
(2006-2018)\end{array}$ & $\begin{array}{c}8307 \text { for } 2006 \\
10,805 \text { for } 2012 \\
9807 \text { for } 2017\end{array}$ & $\begin{array}{l}\text { Polluting fuel: } \\
\text { kerosene, wood, } \\
\text { straw, shrubs, } \\
\text { grass, animal } \\
\text { dung, coal, } \\
\text { charcoal, crop } \\
\text { waste, and others }\end{array}$ & $\begin{array}{l}\text { Clean fuel: LPG, } \\
\text { electricity, biogas, } \\
\text { and natural gas }\end{array}$ & $\begin{array}{l}\text { Pneumonia in } \\
\text { children aged }<5 \text { year: } \\
\text { difficulty in breathing } \\
\text { and chest-related } \\
\text { congestion and } \\
\text { blocked nose and } \\
\text { sought-after treatment }\end{array}$ & $\begin{array}{c}1,2,4,5,14,15,17 \\
20 \text { and } 56\end{array}$ & $\begin{array}{c}\text { Logistic regression } \\
\text { OR, 95\% CI: } \\
\text { PDHS 2006-07: } \\
1.26(0.98,1.61) \\
\text { PDHS 2012-13: } \\
1.16(1.00,1.34) \\
\text { PDHS 2017-18: } \\
1.30(0.99,1.39)\end{array}$ & Moderate \\
\hline $\begin{array}{l}\text { Woolley et al. } \\
\text { (2020) }\end{array}$ & Uganda 2016 & 13,266 & $\begin{array}{l}\text { Households using } \\
\text { wood for cooking }\end{array}$ & $\begin{array}{l}\text { Households using } \\
\text { charcoal for } \\
\text { cooking }\end{array}$ & $\begin{array}{c}\text { ARI in children aged } \\
<5 \text { year: cough and } \\
\text { short rapid } \\
\text { breaths / difficulty } \\
\text { Severe ARI in children } \\
\text { aged }<5 \text { year: cough, } \\
\text { short rapid } \\
\text { breaths / difficulty } \\
\text { breathing and fever }\end{array}$ & $\begin{array}{c}1,1 \mathrm{a}, 1 \mathrm{~b}, 2,3,6,14 \\
15,19,47,49,50 \\
52,53 \text { and } 67\end{array}$ & $\begin{array}{c}\text { Logistic regression } \\
\text { OR, 95\% CI: } \\
\text { ARI: } 1.36 \\
\text { (1.11-1.66) and } \\
\text { Severe ARI: } 1.41 \\
\text { (1.09-1.85), in } \\
\text { wood users } \\
\text { compared to } \\
\text { charcoal users. }\end{array}$ & Moderate \\
\hline
\end{tabular}


Table 1. Cont.

\begin{tabular}{|c|c|c|c|c|c|c|c|c|}
\hline $\begin{array}{c}\text { Authors and } \\
\text { Publication Year }\end{array}$ & $\begin{array}{l}\text { Country and } \\
\text { Survey Year }\end{array}$ & Sample Size & Exposed Group & $\begin{array}{l}\text { Comparator } \\
\text { Group }\end{array}$ & $\begin{array}{l}\text { Outcome: Case } \\
\text { Definition }\end{array}$ & $\begin{array}{c}\text { Covariate } \\
\text { Adjusted (See } \\
\text { Footnote) }\end{array}$ & $\begin{array}{c}\text { Statistical } \\
\text { Method and } \\
\text { Main Results }\end{array}$ & $\begin{array}{c}\text { Risk of Bias } \\
\text { Score }\end{array}$ \\
\hline $\begin{array}{l}\text { Budhathoki et al. } \\
\qquad(2020)\end{array}$ & $\begin{array}{c}\text { Nepal } \\
3 \text { waves } \\
(2006-2016)\end{array}$ & $\begin{array}{l}5139 \text { for } 2011 \\
4887 \text { for } 2016\end{array}$ & $\begin{array}{l}\text { Polluting fuels: } \\
\text { kerosene, wood, } \\
\text { straw, shrubs, } \\
\text { grass, animal } \\
\text { dung, coal and } \\
\text { charcoal }\end{array}$ & $\begin{array}{c}\text { Clean fuels: } \\
\text { electricity, LPG, } \\
\text { biogas and natural } \\
\text { gas }\end{array}$ & $\begin{array}{l}\text { Pneumonia in } \\
\text { children aged <5 year: } \\
\text { cough accompanied } \\
\text { by (1) short, rapid } \\
\text { breathing that is } \\
\text { chest-related and/or } \\
\text { (2) difficult breathing } \\
\text { that is chest-related } \\
\text { (definition from } \\
\text { NDHS report). }\end{array}$ & $\begin{array}{c}4,7,14,15,24,47 \\
49,56\end{array}$ & $\begin{array}{c}\text { Logistic regression } \\
\text { (authors reported } \\
\text { their results as A } \\
\text { relative risk (RR) } \\
\text { RR, 95\% CI: } \\
\text { NDHS 2011: } 1.19 \\
(0.72,1.98) \\
\text { NDHS 2016: } 1.98 \\
(1.01,3.92) \\
\text { Authors declared } \\
\text { that data not } \\
\text { available for the } \\
\text { 2006 NDHS on } \\
\text { fuel use }\end{array}$ & Moderate \\
\hline Rana et al. (2019) & Afghanistan 2015 & 27,565 & $\begin{array}{l}\text { Solid fuel: coal, } \\
\text { lignite, charcoal, } \\
\text { wood, animal } \\
\text { dung, } \\
\text { straw/shrubs/grass, } \\
\text { and kerosene }\end{array}$ & $\begin{array}{l}\text { non-solid fuel: } \\
\text { electricity, LPG, } \\
\text { natural gas, and } \\
\text { biogas }\end{array}$ & $\begin{array}{l}\text { ARI in children aged } \\
<5 \text { year: cough with } \\
\text { shortness of breathing } \\
\text { or difficulty in } \\
\text { breathing }\end{array}$ & $\begin{array}{c}1,2,6,14,15,16 \\
23,51 \text { and } 52\end{array}$ & $\begin{array}{c}\text { Mixed-effect } \\
\text { Poisson regression } \\
\text { Prevalence Ratio, } \\
\text { 95\% CI: } \\
1.10(0.98,1.23)\end{array}$ & Moderate \\
\hline Khan et al., 2018 & $\begin{array}{c}\text { Pakistan, 2012-13 } \\
\text { PDHS }\end{array}$ & 11,040 & $\begin{array}{l}\text { Polluting fuels: } \\
\text { wood, dung, } \\
\text { charcoal, coal, } \\
\text { shrubs/grass/straw, } \\
\text { or kerosene }\end{array}$ & $\begin{array}{c}\text { Cleaner fuel: } \\
\text { natural gas, LPG, } \\
\text { biogas, or } \\
\text { electricity }\end{array}$ & $\begin{array}{l}\text { ARI symptoms in } \\
\text { children aged <5 year: } \\
\text { (1) Cough } \\
\text { accompanied by short } \\
\text { and rapid breathing, } \\
\text { and (2) Cough, } \\
\text { accompanied with } \\
\text { short and rapid } \\
\text { breathing, coupled } \\
\text { with a problem in the } \\
\text { chest }\end{array}$ & $\begin{array}{c}1,2,3,5,6,14,15 \\
16,45 \text { and } 47\end{array}$ & $\begin{array}{l}\text { Logistic regression } \\
\text { OR, 95\% CI: } \\
\text { cough with short } \\
\text { and/or rapid } \\
\text { breathing: } 1.51 \\
(1.03,2.21) \\
\text { cough with short } \\
\text { and/or rapid } \\
\text { breathing and } \\
\text { problem in chest: } \\
1.37(0.84-2.24)\end{array}$ & Moderate \\
\hline
\end{tabular}


Table 1. Cont.

\begin{tabular}{|c|c|c|c|c|c|c|c|c|}
\hline $\begin{array}{c}\text { Authors and } \\
\text { Publication Year }\end{array}$ & $\begin{array}{l}\text { Country and } \\
\text { Survey Year }\end{array}$ & Sample Size & Exposed Group & $\begin{array}{l}\text { Comparator } \\
\text { Group }\end{array}$ & $\begin{array}{l}\text { Outcome: Case } \\
\text { Definition }\end{array}$ & $\begin{array}{c}\text { Covariate } \\
\text { Adjusted (See } \\
\text { Footnote) }\end{array}$ & $\begin{array}{c}\text { Statistical Method } \\
\text { and } \\
\text { Main Results }\end{array}$ & $\begin{array}{c}\text { Risk of Bias } \\
\text { Score }\end{array}$ \\
\hline $\begin{array}{c}\text { Capuno et al. } \\
\text { (2018) }\end{array}$ & $\begin{array}{c}\text { Philippines, } 2013 \\
\text { NDHS }\end{array}$ & 5442 & $\begin{array}{l}\text { Kerosene or solid } \\
\text { fuels (coal, lignite, } \\
\text { charcoal, wood, } \\
\text { crop waste, dung } \\
\text { or } \\
\text { shrubs/grass/straw) }\end{array}$ & $\begin{array}{c}\text { Electricity, LPG, } \\
\text { natural gas or } \\
\text { biogas }\end{array}$ & $\begin{array}{l}\text { ARI Symptoms in } \\
\text { children aged }<5 \text { year: } \\
\text { Cough accompanied } \\
\text { by short, rapid } \\
\text { breathing or difficulty } \\
\text { in breathing as a } \\
\text { result of a problem in } \\
\text { the chest }\end{array}$ & $\begin{array}{c}2,3,4,12,13,14 \\
15,30,31,32,34 \\
35,37,50,52,53 \\
\text { and } 60\end{array}$ & $\begin{array}{c}\text { Logistic regression } \\
\text { with propensity score } \\
\text { marching: Average } \\
\text { treatment effect on the } \\
\text { treated (ATT) } \\
\text { NN1: }-0.024, p<0.1 \\
\text { NN3: }-0.021, p<0.1 \\
\text { NN5: }-0.022, p<0.1\end{array}$ & Critical \\
\hline $\begin{array}{c}\text { Akinyemi et al. } \\
\text { (2018) }\end{array}$ & $\begin{array}{c}\text { Nigeria, 2003-2013 } \\
\text { NDHS }\end{array}$ & $\begin{array}{c}5445(2003) \\
24,975 \\
(2008) \\
28,950(2013)\end{array}$ & $\begin{array}{l}\text { Unclean fuel: coal, } \\
\text { lignite, charcoal, } \\
\text { wood, kerosene, } \\
\text { dung or } \\
\text { shrubs/grass/straw }\end{array}$ & $\begin{array}{c}\text { Clean fuel: } \\
\text { electricity, LPG, } \\
\text { natural gas or } \\
\text { biogas }\end{array}$ & $\begin{array}{l}\text { ARI symptoms in } \\
\text { children aged }<5 \text { year: } \\
\text { Cough in the last } 2 \\
\text { weeks and if a cough } \\
\text { was accompanied by } \\
\text { short rapid breaths }\end{array}$ & $\begin{array}{l}1,3,7,14,15,24 \\
47,49,50,51,52 \\
54,56,57 \text { and } 67\end{array}$ & $\begin{array}{c}\text { Logistic regression } \\
\text { OR, 95\% CI: } \\
0.99(0.98,1.00) \text { for } \\
2003, \\
1.00(0.99,1.01) \text { for } \\
2008, \text { and } \\
1.44(0.66,3.13) \text { for } \\
2013\end{array}$ & Moderate \\
\hline Khan et al. (2017) & $\begin{array}{c}\text { Bangladesh, } \\
\text { 2007-2014 BDHS }\end{array}$ & 22,789 & $\begin{array}{l}\text { Solid fuel: coal, } \\
\text { lignite, charcoal, } \\
\text { wood, dung, } \\
\text { straw/shrubs/grass, } \\
\text { crop waste, and } \\
\text { others }\end{array}$ & $\begin{array}{c}\text { Clean fuel: } \\
\text { electricity, LPG, } \\
\text { natural gas, or } \\
\text { biogas }\end{array}$ & $\begin{array}{l}\text { ARI in children aged } \\
<5 \text { year: Infection in } \\
\text { the nose, trachea or } \\
\text { lungs that interfere } \\
\text { normal breathing }\end{array}$ & $\begin{array}{c}2,6,14,15,50,51 \\
\text { and } 52\end{array}$ & $\begin{array}{c}\text { Logistic regression } \\
\text { OR, } 95 \% \text { CI: } \\
\text { Inside vs. outside } \\
\text { cooking } \\
1.18(1.08,1.33) \\
\text { Clean vs. solid fuel } \\
1.07(0.95,1.20)\end{array}$ & Critical \\
\hline
\end{tabular}


Table 1. Cont.

\begin{tabular}{|c|c|c|c|c|c|c|c|c|}
\hline $\begin{array}{c}\text { Authors and } \\
\text { Publication Year }\end{array}$ & $\begin{array}{l}\text { Country and } \\
\text { Survey Year }\end{array}$ & Sample Size & Exposed Group & $\begin{array}{l}\text { Comparator } \\
\text { Group }\end{array}$ & $\begin{array}{l}\text { Outcome: Case } \\
\text { Definition }\end{array}$ & $\begin{array}{c}\text { Covariate } \\
\text { Adjusted (See } \\
\text { Footnote) }\end{array}$ & $\begin{array}{c}\text { Statistical } \\
\text { Method and } \\
\text { Main Results }\end{array}$ & $\begin{array}{c}\text { Risk of Bias } \\
\text { Score }\end{array}$ \\
\hline Daniel (2016) & $\begin{array}{l}\text { Cameron and } \\
\text { Gabon, } 2011 \\
\text { CDHS and } \\
2012 \text { GDHS }\end{array}$ & $\begin{array}{l}\text { Cameroon } \\
5821 \\
\text { Gabon } \\
1952\end{array}$ & $\begin{array}{l}\text { 1. Only biomass } \\
\text { fuel: wood, crop } \\
\text { waste, dung, } \\
\text { straw/shrubs/grass, } \\
\text { and } \\
\text { 2. Other fuels: } \\
\text { kerosene, coal } \\
\text { (lignite) or } \\
\text { charcoal }\end{array}$ & $\begin{array}{l}\text { Electricity, gas } \\
\text { and/or biomass } \\
\text { fuel }\end{array}$ & $\begin{array}{l}\text { ARI in children aged } \\
<5 \text { year: Cough, } \\
\text { accompanied with } \\
\text { short and rapid } \\
\text { breathing, coupled } \\
\text { with a problem in the } \\
\text { chest }\end{array}$ & $\begin{array}{r}1,7,57,47,50,14 \\
15,45,3,61 \text { and } 52\end{array}$ & $\begin{array}{c}\text { Logistic regression } \\
\text { OR, 95\% CI: } \\
\text { Cameroon: } \\
\text { Only biomass: } \\
5.62 \text { (1.29, } 24.44) \\
\text { Other fuel: } 4.13 \\
(0.51,33.57) \\
\text { Gabon: } \\
\text { Only biomass: } 1.71 \\
(0.98,2.97) \\
\text { Other fuel: } 3.99 \\
(1.46,10.91)\end{array}$ & Critical \\
\hline $\begin{array}{l}\text { Wichmann et al. } \\
\qquad(2015)\end{array}$ & $\begin{array}{c}\text { South Africa, } 1998 \\
\text { SADHS }\end{array}$ & 4679 & $\begin{array}{l}\text { Polluting fuel: } \\
\text { wood, dung, } \\
\text { paraffin, charcoal, } \\
\text { or combination of } \\
\text { these with clean } \\
\text { fuel }\end{array}$ & $\begin{array}{l}\text { Clean fuel: } \\
\text { electricity, LPG or } \\
\text { natural gas, } \\
\text { exclusively }\end{array}$ & $\begin{array}{l}\text { ALRI in children aged } \\
<5 \text { year: Cough, } \\
\text { accompanied by short } \\
\text { and rapid breathing }\end{array}$ & $\begin{array}{c}1,2,3,14,15,31 \\
\text { and } 53\end{array}$ & $\begin{array}{c}\text { OR, 95\% CI: } \\
1.27(1.05,1.55)\end{array}$ & Serious \\
\hline $\begin{array}{c}\text { Buchner et al. } \\
\text { (2015) }\end{array}$ & $\begin{array}{l}\text { SSA countries, } \\
\text { DHS conducted } \\
\text { from } 2000 \text { to } 2011\end{array}$ & 56,437 & $\begin{array}{l}\text { 1. Kerosene } \\
\text { 2. Coal and } \\
\text { charcoal } \\
\text { 3. Wood } \\
\text { 4. Lower-grade } \\
\text { biomass fuels } \\
\text { (shrubs, crop } \\
\text { waste or dung) }\end{array}$ & $\begin{array}{l}\text { Clean fuels: } \\
\text { electricity, gas, } \\
\text { biogas plus } \\
\text { responses of no } \\
\text { food cooked in } \\
\text { house }\end{array}$ & $\begin{array}{l}\text { ALRI in children aged } \\
<5 \text { year: Cough and } \\
\text { short rapid breath or } \\
\text { problems in the chest } \\
\text { or a blocked or } \\
\text { running nose }\end{array}$ & $\begin{array}{c}1,3,6,5,7,16,15 \\
34,48,55,47,45 \\
57,52,60,67,68 \\
30,37 \text { and } 38\end{array}$ & $\begin{array}{c}\text { Mixed model } \\
\text { logistic regression } \\
\text { OR, } 95 \% \text { CI: } \\
\text { Kerosene: } 1.23 \\
(0.77,1.95) \\
\text { Coal/Charcoal: } \\
1.35(1.06,1.72) \\
\text { Wood: } 1.32(1.04, \\
1.66) \\
\text { Lower-grade } \\
\text { biomass: } 1.07 \\
(0.69,1.66)\end{array}$ & Moderate \\
\hline
\end{tabular}


Table 1. Cont

\begin{tabular}{|c|c|c|c|c|c|c|c|c|}
\hline $\begin{array}{c}\text { Authors and } \\
\text { Publication Year }\end{array}$ & $\begin{array}{l}\text { Country and } \\
\text { Survey Year }\end{array}$ & Sample Size & Exposed Group & $\begin{array}{l}\text { Comparator } \\
\text { Group }\end{array}$ & $\begin{array}{l}\text { Outcome: Case } \\
\text { Definition }\end{array}$ & $\begin{array}{l}\text { Covariate } \\
\text { Adjusted (See } \\
\text { Footnote) }\end{array}$ & $\begin{array}{c}\text { Statistical } \\
\text { Method and } \\
\text { Main Results }\end{array}$ & $\begin{array}{c}\text { Risk of Bias } \\
\text { Score }\end{array}$ \\
\hline $\begin{array}{l}\text { Acharya et al. } \\
\text { (2015) }\end{array}$ & $\begin{array}{l}\text { Nepal, } 2011 \\
\text { NDHS }\end{array}$ & 4773 & $\begin{array}{l}\text { Solid fuel: wood, } \\
\text { animal dung, } \\
\text { straw, shrubs, } \\
\text { grass, crop waste, } \\
\text { coal (lignite) or } \\
\text { charcoal }\end{array}$ & $\begin{array}{l}\text { Cleaner fuel: LPG, } \\
\text { biogas, electricity, } \\
\text { natural gas or } \\
\text { kerosene }\end{array}$ & $\begin{array}{l}\text { ARI in children aged } \\
<5 \text { year: Cough } \\
\text { accompanied by } \\
\text { short/rapid breath } \\
\text { and problem in chest }\end{array}$ & $\begin{array}{l}1,2,3,15,16,50 \\
51,52,53 \text { and } 65\end{array}$ & $\begin{array}{c}\text { Logistic regression } \\
\text { OR, 95\% CI: } \\
1.79(1.02,3.14) .\end{array}$ & Serious \\
\hline Patel et al. (2013) & $\begin{array}{l}\text { India, INFHS } \\
\text { conducted from } \\
1992 \text { to } 2006\end{array}$ & 36,254 & $\begin{array}{c}\text { 1. Highly } \\
\text { polluting fuel: } \\
\text { wood, crop waste, } \\
\text { dung, or straw } \\
\text { 2. Medium } \\
\text { polluting fuel: } \\
\text { coal/lignite, } \\
\text { charcoal, or } \\
\text { kerosene }\end{array}$ & $\begin{array}{l}\text { Low polluting } \\
\text { fuel: LPG, natural } \\
\text { gas or electricity }\end{array}$ & $\begin{array}{l}\text { ALRI in children aged } \\
0-35 \text { months: Cough } \\
\text { accompanied by rapid } \\
\text { breathing }\end{array}$ & $\begin{array}{c}14,15,24,1,2,4 \\
52,31,50,51 \text { and } \\
25\end{array}$ & $\begin{array}{c}\text { Logistic regression } \\
\text { OR, } 95 \% \text { CI: } \\
\text { Highly polluting } \\
\text { fuels: } \\
1.48(1.08,2.03) \text { for } \\
1992 \\
1.54(1.33,1.77) \text { for } \\
1999 \\
1.53(1.21,1.93) \text { for } \\
2006 \\
\text { Medium polluting } \\
\text { fuels: } \\
1.39(1.01,1.92) \text { for } \\
1992 \\
1.47(1.22,1.76) \text { for } \\
1999 \\
1.31(0.92,1.88) \text { for } \\
2006\end{array}$ & Critical \\
\hline $\begin{array}{l}\text { Kilabuko et al. } \\
\qquad(2007)\end{array}$ & $\begin{array}{l}\text { Tanzania, } \\
\text { 2004-2005 } \\
\text { TDHS }\end{array}$ & 5224 & $\begin{array}{l}\text { Biomass fuels: } \\
\text { firewood, straw, } \\
\text { dung or crop } \\
\text { waste }\end{array}$ & $\begin{array}{l}\text { Charcoal or } \\
\text { kerosene }\end{array}$ & $\begin{array}{l}\text { ARI in children aged } \\
<5 \text { year: Cough } \\
\text { together with short } \\
\text { and rapid breathing }\end{array}$ & $\begin{array}{c}14,15,1,2,50,51 \\
\text { and } 52\end{array}$ & $\begin{array}{c}\text { Logistic regression } \\
\text { OR, 95\% CI: } \\
1.01(0.78,1.42)\end{array}$ & Critical \\
\hline
\end{tabular}


Table 1. Cont.

\begin{tabular}{|c|c|c|c|c|c|c|c|c|}
\hline $\begin{array}{c}\text { Authors and } \\
\text { Publication Year }\end{array}$ & $\begin{array}{l}\text { Country and } \\
\text { Survey Year }\end{array}$ & Sample Size & Exposed Group & $\begin{array}{l}\text { Comparator } \\
\text { Group }\end{array}$ & $\begin{array}{l}\text { Outcome: Case } \\
\text { Definition }\end{array}$ & $\begin{array}{c}\text { Covariate } \\
\text { Adjusted (See } \\
\text { Footnote) }\end{array}$ & $\begin{array}{c}\text { Statistical } \\
\text { Method and } \\
\text { Main Results }\end{array}$ & $\begin{array}{c}\text { Risk of Bias } \\
\text { Score }\end{array}$ \\
\hline $\begin{array}{l}\text { Mishra et al. } \\
\text { (2005) }\end{array}$ & $\begin{array}{c}\text { India, 1998-1999 } \\
\text { NFHS }\end{array}$ & 29,768 & $\begin{array}{l}\text { 1. Biomass fuels: } \\
\text { wood, crop waste, } \\
\text { or dung } \\
\text { 2. Mixed fuels: } \\
\text { biomass fuels, } \\
\text { charcoal and coal }\end{array}$ & $\begin{array}{c}\text { Cleaner fuels: } \\
\text { electricity, LPG, } \\
\text { biogas or kerosene }\end{array}$ & $\begin{array}{l}\text { ARI in children aged } \\
0-36 \text { months: Cough, } \\
\text { with breath faster than } \\
\text { usual with short, } \\
\text { rapid breaths }\end{array}$ & $\begin{array}{c}1,2,3,14,15,30 \\
31,7,49,57,47,45 \\
52,50,51 \text { and } 53\end{array}$ & $\begin{array}{l}\text { Logistic regression } \\
\text { OR, } 95 \% \text { CI: } \\
\text { Biomass fuel: } 1.58 \\
(1.28,1.95) \\
\text { Mixed fuel: } 1.41 \\
(1.17,1.70)\end{array}$ & Serious \\
\hline Mishra (2003) & $\begin{array}{c}\text { Zimbabwe, } 1999 \\
\text { ZDHS }\end{array}$ & 3559 & $\begin{array}{c}\text { 1. Highly } \\
\text { polluting fuels: } \\
\text { wood, dung, or } \\
\text { straw } \\
\text { 2. Medium } \\
\text { polluting fuels: } \\
\text { kerosene or } \\
\text { charcoal }\end{array}$ & $\begin{array}{l}\text { Low polluting } \\
\text { fuels: LPG, natural } \\
\text { gas, or electricity }\end{array}$ & $\begin{array}{l}\text { ARI in children aged } \\
<5 \text { year: Cough } \\
\text { accompanied by short } \\
\text { and rapid breathing }\end{array}$ & $\begin{array}{c}1,2,3,7,14,15,30 \\
51 \text { and } 52\end{array}$ & $\begin{array}{l}\text { Logistic regression } \\
\text { OR, 95\% CI: } \\
\text { High polluted: } \\
2.20(1.16,4.19) \\
\text { Medium polluted: } \\
1.33(0.64,2.77)\end{array}$ & Critical \\
\hline
\end{tabular}

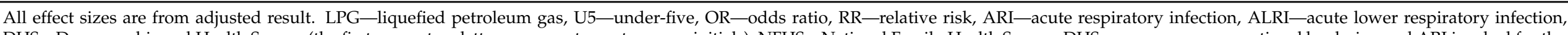

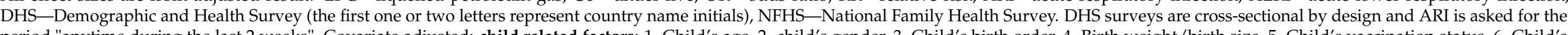

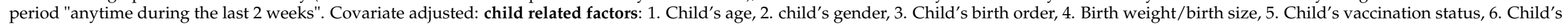

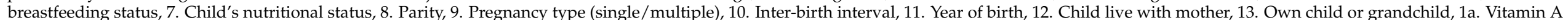

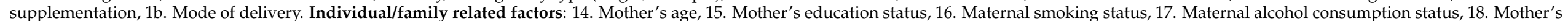

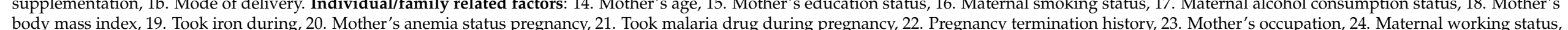

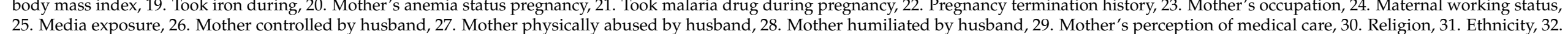

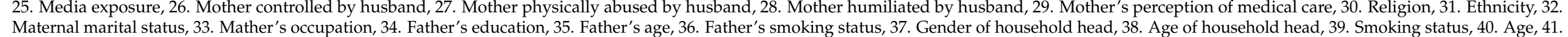

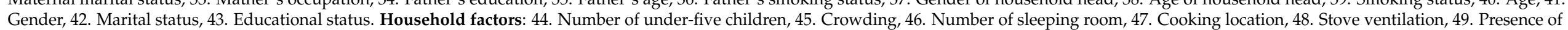

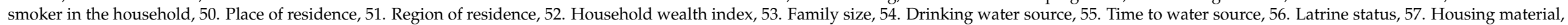

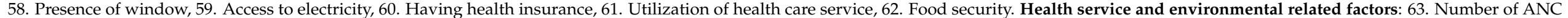

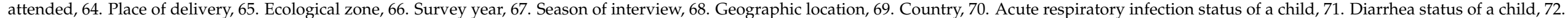

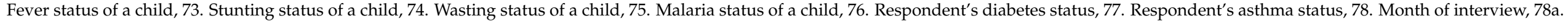
History of TB contact. 
Table 2. DHS data-based evidence on the association between cooking fuel use and under-five mortality (all-cause).

\begin{tabular}{|c|c|c|c|c|c|c|c|c|}
\hline $\begin{array}{c}\text { Authors and } \\
\text { Publication Year }\end{array}$ & $\begin{array}{l}\text { Country and } \\
\text { Survey Year }\end{array}$ & Sample Size & Exposed Group & $\begin{array}{l}\text { Comparator } \\
\text { Group }\end{array}$ & Case Definition & $\begin{array}{c}\text { Covariate } \\
\text { Adjusted (Table } 1 \\
\text { Footnote) }\end{array}$ & $\begin{array}{c}\text { Statistical } \\
\text { Method and } \\
\text { Main Results }\end{array}$ & Risk of BiasScore \\
\hline $\begin{array}{l}\text { Samuel et al. } \\
\text { (2018) }\end{array}$ & $\begin{array}{c}\text { Nigeria, } 2013 \\
\text { NDHS }\end{array}$ & 10,983 & $\begin{array}{l}\text { Solid fuel: wood, } \\
\text { charcoal and dung, } \\
\text { in a kitchen inside } \\
\text { the house }\end{array}$ & $\begin{array}{l}\text { Non-solid fuel: } \\
\text { electric, gas and } \\
\text { kerosene, in a } \\
\text { kitchen inside the } \\
\text { house }\end{array}$ & $\begin{array}{l}\text { Under-five mortality } \\
\text { during the last five } \\
\text { years preceding the } \\
\text { survey }\end{array}$ & $15,52,50$ and 51 & $\begin{array}{c}\text { Logistic regression } \\
\text { OR, 95\% CI: } \\
1.23(0.98,1.54)\end{array}$ & Critical \\
\hline Nisha et al. (2018) & $\begin{array}{c}\text { Bangladesh, 2004, } \\
\text { 2007, 2011, and } \\
2014 \text { BDHS }\end{array}$ & 35,052 & $\begin{array}{c}\text { Polluting fuels: } \\
\text { kerosene, } \\
\text { coal/lignite, } \\
\text { charcoal, wood, } \\
\text { crop waste, dung } \\
\text { or } \\
\text { straw/shrubs/grass }\end{array}$ & $\begin{array}{c}\text { Clean fuels: } \\
\text { electricity, LPG, } \\
\text { natural gas, and } \\
\text { biogas }\end{array}$ & $\begin{array}{l}\text { Death between the } \\
\text { ages of } 0 \text { and } 6 \text { days }\end{array}$ & $\begin{array}{c}3,14,15,18,24,50 \\
52,47 \text { and } 66\end{array}$ & $\begin{array}{l}\text { Logistic regression } \\
\text { OR 95\% CI: } \\
\text { Early neonatal: } \\
1.46(1.01,2.10)\end{array}$ & Critical \\
\hline Naz et al. (2018) & $\begin{array}{c}\text { Nepal, 2001-2011 } \\
\text { NDHS }\end{array}$ & 17,780 & $\begin{array}{l}\text { Polluting fuels: } \\
\text { kerosene, } \\
\text { coal/lignite, } \\
\text { charcoal, wood, } \\
\text { straw/shrubs/grass, } \\
\text { crop waste or } \\
\text { dung }\end{array}$ & $\begin{array}{c}\text { Clean fuels: } \\
\text { electricity, LPG, } \\
\text { natural gas and } \\
\text { biogas }\end{array}$ & $\begin{array}{c}\text { Under-five mortality } \\
\text { within } 5 \text { years prior to } \\
\text { the survey }\end{array}$ & $\begin{array}{r}2,6,14,15,24,50 \\
52,47,65,57 \text { and } 8\end{array}$ & $\begin{array}{c}\text { Logistic regression } \\
\text { OR, 95\% CI: } \\
\text { Neonatal: } 2.67 \\
(1.47,4.82) \\
\text { Post-neonatal: } 1.61 \\
(0.67,3.87) \\
\text { Child: } 1.29(0.33, \\
4.99) \\
\text { Under-five: } 2.19 \\
(1.37,3.51)\end{array}$ & Moderate \\
\hline Owili et al. (2017) & $\begin{array}{l}23 \text { SSA countries, } \\
\text { DHS conducted } \\
\text { from } 2010 \text { to } 2014\end{array}$ & 783,691 & $\begin{array}{l}\text { 1. Charcoal } \\
\text { 2. Other biomass: } \\
\text { wood, } \\
\text { straw/shrubs/grass, } \\
\text { crop waste, or } \\
\text { dung } \\
\text { 3. Other polluting } \\
\text { fuel: coal/lignite } \\
\text { or kerosene }\end{array}$ & $\begin{array}{c}\text { Clean fuel: } \\
\text { electricity, natural } \\
\text { gas, biogas or LPG }\end{array}$ & $\begin{array}{c}\text { All-cause mortality of } \\
\text { under-five children } \\
\text { within } 5 \text { years prior to } \\
\text { the survey }\end{array}$ & $\begin{array}{c}47,69,50,2,6,14 \\
15,53,44,52,49 \\
33 \text { and } 23\end{array}$ & $\begin{array}{c}\text { Cox regression } \\
\text { HR, 95\% CI: } \\
\text { Charcoal: } 1.21 \\
(1.10,1.34) \\
\text { Other biomass: } \\
1.20(1.08,1.32) \\
\text { Other fuel: } 1.01 \\
(0.90,1.14)\end{array}$ & Moderate \\
\hline
\end{tabular}


Table 2. Cont.

\begin{tabular}{|c|c|c|c|c|c|c|c|c|}
\hline $\begin{array}{c}\text { Authors and } \\
\text { Publication Year }\end{array}$ & $\begin{array}{l}\text { Country and } \\
\text { Survey Year }\end{array}$ & Sample Size & Exposed Group & $\begin{array}{l}\text { Comparator } \\
\text { Group }\end{array}$ & Case Definition & $\begin{array}{c}\text { Covariate } \\
\text { Adjusted (Table } 1 \\
\text { Footnote) }\end{array}$ & $\begin{array}{c}\text { Statistical } \\
\text { Method and } \\
\text { Main Results }\end{array}$ & Risk of BiasScore \\
\hline Naz et al. (2017) & $\begin{array}{c}\text { Pakistan, } 2013 \\
\text { PDHS }\end{array}$ & 11,507 & $\begin{array}{l}\text { Polluting fuels: } \\
\text { kerosene, } \\
\text { coal/lignite, } \\
\text { charcoal, wood, } \\
\text { straw/shrubs/grass } \\
\text { or dung }\end{array}$ & $\begin{array}{c}\text { Clean fuels: } \\
\text { electricity, LPG, } \\
\text { natural gas and } \\
\text { biogas }\end{array}$ & $\begin{array}{l}\text { Neonatal, } \\
\text { post-neonatal, } \\
\text { child and } \\
\text { under-five } \\
\text { mortality }\end{array}$ & $\begin{array}{c}1,2,6,14,15,24 \\
16,50,52,57 \text { and } \\
47\end{array}$ & $\begin{array}{c}\text { Logistic regression } \\
\text { OR, } 95 \% \text { CI: } \\
\text { Logistic regression } \\
\text { Neonatal: } 1.09 \\
(0.77,1.54) \\
\text { Post-neonatal: } 1.31 \\
(0.75,2.27) \\
\text { Child: } 1.98(0.75, \\
5.25) \\
\text { Under-five: } 1.22 \\
(0.92,1.64)\end{array}$ & Moderate \\
\hline Khan et al. (2017) & $\begin{array}{c}\text { Bangladesh, } \\
\text { 2007-2014 BDHS }\end{array}$ & 22,789 & $\begin{array}{l}\text { Solid fuels: coal, } \\
\text { lignite, charcoal, } \\
\text { wood, } \\
\text { straw/shrubs/grass, } \\
\text { crop waste, dung } \\
\text { and others }\end{array}$ & $\begin{array}{c}\text { Clean fuel: } \\
\text { electricity, LPG, } \\
\text { natural gas, and } \\
\text { biogas }\end{array}$ & $\begin{array}{l}\text { Neonatal, infant } \\
\text { and under-five } \\
\text { mortality }\end{array}$ & $\begin{array}{c}2,6,14,15,50,51 \\
\text { and } 52\end{array}$ & $\begin{array}{c}\text { Logistic regression } \\
\text { OR, } 95 \% \text { CI: } \\
\text { Neonatal: } 1.23 \\
(0.97,1.55) \\
\text { Infant: } 1.15(0.94 \\
1.42) \\
\text { Under-five: } 1.11 \\
(0.91,1.35)\end{array}$ & Serious \\
\hline Naz et al. (2016) & $\begin{array}{c}\text { India, 1992-2006 } \\
\text { NFHS }\end{array}$ & 166,382 & $\begin{array}{l}\text { polluting fuels: } \\
\text { kerosene, } \\
\text { coal/lignite, } \\
\text { charcoal, wood, } \\
\text { straw/shrubs/grass, } \\
\text { crop waste or } \\
\text { dung }\end{array}$ & $\begin{array}{c}\text { Clean fuels: } \\
\text { electricity, LPG, } \\
\text { natural gas and } \\
\text { biogas }\end{array}$ & $\begin{array}{l}\text { Neonatal, } \\
\text { post-neonatal, } \\
\text { child and } \\
\text { under-five } \\
\text { mortality }\end{array}$ & $\begin{array}{c}1,6,14,15,24,16 \\
50,52,57,66 \text { and } \\
47\end{array}$ & $\begin{array}{c}\text { Logistic regression } \\
\text { OR, } 95 \% \text { CI: } \\
\text { Neonatal: } 1.23 \\
(1.09,1.39) \\
\text { Post-neonatal: } 1.42 \\
(1.19,1.71) \\
\text { Child: } 1.42(1.05, \\
1.91) \\
\text { Under-five: } 1.30 \\
(1.18,1.43)\end{array}$ & Moderate \\
\hline
\end{tabular}


Table 2. Cont.

\begin{tabular}{|c|c|c|c|c|c|c|c|c|}
\hline $\begin{array}{c}\text { Authors and } \\
\text { Publication Year }\end{array}$ & $\begin{array}{l}\text { Country and } \\
\text { Survey Year }\end{array}$ & Sample Size & Exposed Group & $\begin{array}{l}\text { Comparator } \\
\text { Group }\end{array}$ & Case Definition & $\begin{array}{c}\text { Covariate } \\
\text { Adjusted (Table } 1 \\
\text { Footnote) }\end{array}$ & $\begin{array}{c}\text { Statistical } \\
\text { Method and } \\
\text { Main Results }\end{array}$ & Risk of BiasScore \\
\hline $\begin{array}{c}\text { Akinyemi et al. } \\
(2016)\end{array}$ & $\begin{array}{l}15 \text { SSA countries, } \\
\text { DHS conducted } \\
\text { from } 2010 \text { to } 2014\end{array}$ & 143,602 & $\begin{array}{l}\text { Solid fuel: coal, } \\
\text { lignite, charcoal, } \\
\text { wood, } \\
\text { straw/shrubs/grass, } \\
\text { crop waste or } \\
\text { dung }\end{array}$ & $\begin{array}{l}\text { Non-solid fuel: } \\
\text { electricity, LPG, } \\
\text { natural gas, biogas } \\
\text { and kerosene }\end{array}$ & $\begin{array}{c}\text { Infant and child } \\
\text { mortality }\end{array}$ & $\begin{array}{l}2,3,4,10,14,15 \\
23,16,25,49,50 \\
\quad 52 \text { and } 53\end{array}$ & $\begin{array}{c}\text { Cox regression } \\
\text { HR, 95\% CI: } \\
\text { Smoking + solid } \\
\text { fuel: } \\
\text { 1.59 }(1.26,1.99) \\
\text { Smoking + } \\
\text { non-solid fuel: } \\
0.86(0.44,1.68) \\
\text { No smoking + } \\
\text { solid fuel: } \\
1.44(1.18,1.76)\end{array}$ & Serious \\
\hline Naz et al. (2015) & $\begin{array}{c}\text { Bangladesh, 2004, } \\
2007 \text { and } 2011 \\
\text { BDHS }\end{array}$ & 18,308 & $\begin{array}{l}\text { Polluting fuels: } \\
\text { kerosene, } \\
\text { coal/lignite, } \\
\text { charcoal, wood, } \\
\text { straw/shrubs/grass, } \\
\text { crop waste or } \\
\text { dung }\end{array}$ & $\begin{array}{c}\text { Clean fuels: } \\
\text { electricity, LPG, } \\
\text { natural gas, and } \\
\text { biogas }\end{array}$ & $\begin{array}{c}\text { Neonatal, infant } \\
\text { and under-five } \\
\text { mortality }\end{array}$ & $\begin{array}{c}6,14,15,24,52,50 \\
57 \text { and } 66\end{array}$ & $\begin{array}{c}\text { Logistic regression } \\
\text { OR, 95\% CI: } \\
\text { Neonatal: } 1.49 \\
(1.01,2.22) \\
\text { Infant: } 1.27(0.91, \\
1.77) \\
\text { Under-five: } 1.14 \\
(0.83,1.55)\end{array}$ & Moderate \\
\hline $\begin{array}{l}\text { Kleimola et al. } \\
\text { (2015) }\end{array}$ & $\begin{array}{l}47 \text { Countries, DHS } \\
\text { conducted from } \\
2001 \text { to } 2012\end{array}$ & $\begin{array}{c}774,638 \text { neonates } \\
\text { and } \\
751,571 \text { children }\end{array}$ & $\begin{array}{l}\text { Kerosene and } \\
\text { Solid fuels: coal, } \\
\text { charcoal, and } \\
\text { biomass such as } \\
\text { wood, crop waste } \\
\text { or dung }\end{array}$ & $\begin{array}{c}\text { Clean fuels: } \\
\text { electricity, LPG, } \\
\text { natural gas, and } \\
\text { biogas }\end{array}$ & $\begin{array}{l}\text { Neonatal and } \\
\text { child mortality }\end{array}$ & $\begin{array}{c}2,3,14,15,16,52 \\
50 \text { and } 69\end{array}$ & $\begin{array}{c}\text { Poisson regression } \\
\text { RR, 95\% CI: } \\
\text { Kerosene- } \\
\text { neonatal: } 1.34 \\
(1.18,1.52) \\
\text { solid fuel- } \\
\text { neonatal: } 1.24 \\
(1.14,1.34) \\
\text { Kerosene- child: } \\
1.12(0.99,1.27) \\
\text { Solid fuel- child: } \\
1.21(1.12,1.30)\end{array}$ & Critical \\
\hline
\end{tabular}


Table 2. Cont.

\begin{tabular}{|c|c|c|c|c|c|c|c|c|}
\hline $\begin{array}{c}\text { Authors and } \\
\text { Publication Year }\end{array}$ & $\begin{array}{l}\text { Country and } \\
\text { Survey Year }\end{array}$ & Sample Size & Exposed Group & $\begin{array}{l}\text { Comparator } \\
\text { Group }\end{array}$ & Case Definition & $\begin{array}{c}\text { Covariate } \\
\text { Adjusted (Table } 1 \\
\text { Footnote) }\end{array}$ & $\begin{array}{c}\text { Statistical } \\
\text { Method and } \\
\text { Main Results }\end{array}$ & Risk of BiasScore \\
\hline Ezeh et al. (2014) & $\begin{array}{l}\text { Nigeria, } 2013 \\
\text { NDHS }\end{array}$ & 30,726 & $\begin{array}{c}\text { Solid fuel: } \\
\text { coal/lignite, } \\
\text { charcoal, wood, } \\
\text { straw/shrubs/grass, } \\
\text { crop waste and } \\
\text { dung }\end{array}$ & $\begin{array}{l}\text { Non-solid fuels: } \\
\text { electricity, LPG, } \\
\text { natural gas, } \\
\text { biogas, or } \\
\text { kerosene }\end{array}$ & $\begin{array}{c}\text { Neonatal, } \\
\text { post-neonatal and } \\
\text { child mortality }\end{array}$ & $\begin{array}{l}2,6,4,14,15,24 \\
50 \text { and } 47\end{array}$ & $\begin{array}{c}\text { Cox regression } \\
\text { HR, 95\% CI: } \\
\text { Neonatal: } 1.01 \\
(0.73,1.26) \\
\text { Post-neonatal: } 1.92 \\
(1.42,2.58) \\
\text { Child: } 1.63 \text { (1.09, } \\
2.42)\end{array}$ & Serious \\
\hline $\begin{array}{l}\text { Pandey et al. } \\
\text { (2013) }\end{array}$ & $\begin{array}{l}\text { India, 2005-6 } \\
\text { NFHS }\end{array}$ & 25,839 & $\begin{array}{l}\text { Solid fuel: } \\
\text { coal/lignite, } \\
\text { charcoal, wood, } \\
\text { straw, shrubs, } \\
\text { grass, crop waste, } \\
\text { or dung }\end{array}$ & $\begin{array}{c}\text { Other fuels: } \\
\text { electricity, LPG, } \\
\text { natural gas, biogas } \\
\text { or kerosene }\end{array}$ & $\begin{array}{l}\text { Women who had } \\
\text { experienced death } \\
\text { of at least one } \\
\text { under-five child } \\
\text { death }\end{array}$ & $\begin{array}{c}16,14,15,30,26 \\
27,28,35,34,52 \\
50,48 \text { and } 58\end{array}$ & $\begin{array}{c}\text { Logistic regression } \\
\text { OR, 95\% CI: } \\
1.23(1.06,1.43)\end{array}$ & Serious \\
\hline $\begin{array}{l}\text { Epstein et al. } \\
\quad(2013)\end{array}$ & $\begin{array}{l}\text { India, 2005-6 } \\
\text { NFHS }\end{array}$ & 14,850 & $\begin{array}{c}\text { High-pollution } \\
\text { fuels: } \\
\text { (a) kerosene, } \\
\text { (b) coal/coal } \\
\text { lignite, } \\
\text { (c) biomass fuels } \\
\text { (wood, charcoal, } \\
\text { crop waste, dung } \\
\text { or } \\
\text { straw / shrubs/grass) }\end{array}$ & $\begin{array}{l}\text { Low-pollution } \\
\text { fuels: LPG, natural } \\
\text { gas or biogas }\end{array}$ & $\begin{array}{l}\text { Death in the first } \\
0-28 \text { days of life }\end{array}$ & $\begin{array}{c}2,4,14,15,30,16 \\
23,18,50,51,59 \\
64,57,54,29,63,8 \\
\text { and } 10\end{array}$ & $\begin{array}{c}\text { Logistic regression } \\
\text { OR, 95\% CI: } \\
\text { Kerosene: } 2.88 \\
(1.18,7.02) \\
\text { Biomass: } 0.84 \\
(0.39,1.81) \\
\text { Coal: } 24.15(7.98, \\
73.12)\end{array}$ & Moderate \\
\hline $\begin{array}{l}\text { Wichmann et al. } \\
\text { (2006) }\end{array}$ & $\begin{array}{l}\text { South Africa, } 1998 \\
\text { SADHS }\end{array}$ & 3556 & $\begin{array}{l}\text { Polluting fuels: } \\
\text { wood, dung, coal } \\
\text { or paraffin }\end{array}$ & $\begin{array}{l}\text { Clean fuels: LPG, } \\
\text { natural gas or } \\
\text { electricity, } \\
\text { exclusively }\end{array}$ & $\begin{array}{l}\text { Mortality during } \\
1-59 \text { months of } \\
\text { age }\end{array}$ & $\begin{array}{c}1,2,3,6,7,10,14 \\
54,56,45 \text { and } 11\end{array}$ & $\begin{array}{l}\text { Cox regression } \\
\text { HR, 95\% CI: } \\
1.99(1.04,3.68)\end{array}$ & Moderate \\
\hline
\end{tabular}

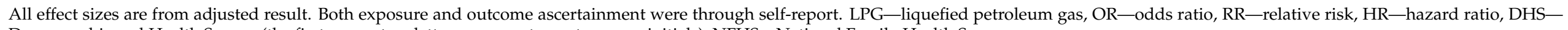
Demographic and Health Survey (the first one or two letters represent country name initials), NFHS—National Family Health Survey. 
Table 3. DHS data-based evidence on the association between cooking fuel use and under-five nutritional status.

\begin{tabular}{|c|c|c|c|c|c|c|c|c|c|}
\hline $\begin{array}{c}\text { Authors and } \\
\text { Publication } \\
\text { Year }\end{array}$ & $\begin{array}{l}\text { Country and } \\
\text { Survey Year }\end{array}$ & $\begin{array}{l}\text { Unit of } \\
\text { Analyses }\end{array}$ & Sample Size & Exposed Group & $\begin{array}{l}\text { Comparator } \\
\text { Group }\end{array}$ & $\begin{array}{l}\text { Outcome } \\
\text { Definition }\end{array}$ & $\begin{array}{c}\text { Covariate } \\
\text { Adjusted (Table } 1 \\
\text { Footnote) }\end{array}$ & $\begin{array}{c}\text { Statistical Method } \\
\text { and } \\
\text { Main Results }\end{array}$ & $\begin{array}{c}\text { Risk of Bias } \\
\text { Score }\end{array}$ \\
\hline $\begin{array}{l}\text { Dadras et al. } \\
\text { (2017) }\end{array}$ & $\begin{array}{l}\text { Nepal, } 2011 \\
\text { NDHS }\end{array}$ & $\begin{array}{l}\text { Under-five } \\
\text { children }\end{array}$ & 2262 & $\begin{array}{l}\text { High polluting } \\
\text { fuels: wood, } \\
\text { dung, straw or } \\
\text { crop waste }\end{array}$ & $\begin{array}{l}\text { Low polluting } \\
\text { fuels: LPG, } \\
\text { natural gas, } \\
\text { biogas, } \\
\text { electricity, } \\
\text { kerosene, coal or } \\
\text { charcoal }\end{array}$ & $\begin{array}{l}\text { Stunting } \\
\text { height-for age } \\
\text { Z-score } \\
<-2 \text { SD }\end{array}$ & $\begin{array}{c}1,3,4,15,16,18 \\
47,51,52,54,31 \\
\text { and } 62\end{array}$ & $\begin{array}{c}\text { Logistic regression } \\
\text { OR, 95\% CI: } \\
1.13(0.72,1.76)\end{array}$ & Critical \\
\hline $\begin{array}{l}\text { Machisa et al. } \\
\text { (2013) }\end{array}$ & $\begin{array}{l}\text { Swaziland, } \\
\text { 2006-7 SDHS }\end{array}$ & $\begin{array}{l}\text { Children aged } \\
6-36 \text { months }\end{array}$ & 1150 & $\begin{array}{l}\text { Biomass fuel: } \\
\text { coal, charcoal or } \\
\text { wood with or } \\
\text { without cleaner } \\
\text { fuels }\end{array}$ & $\begin{array}{l}\text { Cleaner fuels: } \\
\text { LPG, natural } \\
\text { gas, electricity } \\
\text { and/or paraffin, } \\
\text { exclusively }\end{array}$ & $\begin{array}{l}\text { Anaemia and } \\
\text { stunting in } \\
\text { under } 5 \text { children }\end{array}$ & $\begin{array}{c}1,2,3,10,4,14,15 \\
18,19,20,50,51 \\
47,52,70,71 \text { and } \\
72\end{array}$ & $\begin{array}{c}\text { Logistic regression } \\
\text { (multinomial) } \\
\text { RRR, 95\% CI: } \\
\text { Mild stunting: } 1.1 \\
(0.6,2.0) \\
\text { Sever stunting: } 1.4 \\
(0.7,2.7) \\
\text { Only descriptive } \\
\text { result reported for } \\
\text { anaemia }\end{array}$ & Serious \\
\hline Kyu et al. (2010) & $\begin{array}{l}\text { DHS conducted } \\
\text { from } 2003 \text { to } \\
2007 \text { in } 29 \\
\text { countries }\end{array}$ & $\begin{array}{l}\text { Children aged } \\
0-59 \text { months }\end{array}$ & 117,454 & $\begin{array}{l}\text { Households } \\
\text { using wood, } \\
\text { straw, dung or } \\
\text { crop waste }\end{array}$ & $\begin{array}{l}\text { Households } \\
\text { using Electricity, } \\
\text { natural gas, } \\
\text { biogas or } \\
\text { kerosene }\end{array}$ & $\begin{array}{l}\text { Anaemia in } \\
\text { children }\end{array}$ & $\begin{array}{c}1,2,14,15,16,19 \\
20,52,69,71,72 \\
44,73 \text { and } 74\end{array}$ & $\begin{array}{c}\text { Logistic regression } \\
\text { (multinomial) } \\
\text { RRR, 95\% CI: } \\
\text { Mild: } 1.07(1.01, \\
1.13) \\
\text { Moderate/sever: } \\
0.99(0.94,1.05) \\
\end{array}$ & Serious \\
\hline Kyu et al. (2009) & $\begin{array}{l}\text { DHS conducted } \\
\text { from } 2005 \text { to } \\
2007 \text { in } 7 \\
\text { countries }\end{array}$ & $\begin{array}{l}\text { Under-five } \\
\text { children }\end{array}$ & 28,439 & $\begin{array}{l}\text { Coal and } \\
\text { biomass fuels } \\
\text { such as wood, } \\
\text { straw or dung) }\end{array}$ & $\begin{array}{l}\text { Cleaner fuels: } \\
\text { electricity, } \\
\text { natural gas, } \\
\text { biogas and } \\
\text { kerosene }\end{array}$ & Stunting & $\begin{array}{c}1,2,4,6,14,15,16 \\
36,44,52 \text { and } 69\end{array}$ & $\begin{array}{c}\text { Logistic regression } \\
\text { (multinomial) } \\
\text { RRR, } 95 \% \text { CI: } \\
\text { Stunting: } 1.25 \text { (1.08, } \\
1.44) \\
\text { Severe stunting: } \\
1.27(1.02,1.59)\end{array}$ & Serious \\
\hline
\end{tabular}


Table 3. Cont.

\begin{tabular}{|c|c|c|c|c|c|c|c|c|c|}
\hline $\begin{array}{l}\text { Authors and } \\
\text { Publication } \\
\text { Year }\end{array}$ & $\begin{array}{l}\text { Country and } \\
\text { Survey Year }\end{array}$ & $\begin{array}{l}\text { Unit of } \\
\text { Analyses }\end{array}$ & Sample Size & Exposed Group & $\begin{array}{l}\text { Comparator } \\
\text { Group }\end{array}$ & $\begin{array}{l}\text { Outcome } \\
\text { Definition }\end{array}$ & $\begin{array}{c}\text { Covariate } \\
\text { Adjusted (Table } 1 \\
\text { Footnote) }\end{array}$ & $\begin{array}{c}\text { Statistical Method } \\
\text { and } \\
\text { Main Results }\end{array}$ & $\begin{array}{c}\text { Risk of Bias } \\
\text { Score }\end{array}$ \\
\hline $\begin{array}{l}\text { Mishra et al. } \\
\text { (2007) }\end{array}$ & $\begin{array}{c}\text { India, 1998-99 } \\
\text { NFHS }\end{array}$ & $\begin{array}{l}\text { Children aged } \\
0-35 \text { months }\end{array}$ & 29,768 & $\begin{array}{l}\text { 1. only biofuels } \\
\text { (wood, crop } \\
\text { waste, or dung) } \\
\text { 2. mix of } \\
\text { biomass fuels } \\
\text { and cleaner } \\
\text { fuels } \\
\text { (coal/lignite or } \\
\text { charcoal) }\end{array}$ & $\begin{array}{l}\text { Cleaner fuels: } \\
\text { electricity, liquid } \\
\text { petroleum gas, } \\
\text { biogas, or } \\
\text { kerosene }\end{array}$ & $\begin{array}{l}\text { Stunting and } \\
\text { anaemia }\end{array}$ & $\begin{array}{c}2,3,14,15,18,20 \\
19,30,31,70,71 \\
75,57,51,52,45 \\
\text { and } 49\end{array}$ & $\begin{array}{c}\text { Logistic regression } \\
\text { (multinomial) } \\
\text { RRR, 95\% CI: } \\
\text { Moderate-to-severe } \\
\text { anaemia } \\
\text { Only biomass fuel: } \\
1.58(1.28,1.94) \\
\text { Mixed fuel: } 1.36 \\
(1.13,1.63) \\
\text { Severe stunting } \\
\text { Only biomass fuel: } \\
1.90(1.49,2.42) \\
\text { Mixed fuel: } 1.26 \\
(1.00,1.58)\end{array}$ & Serious \\
\hline
\end{tabular}

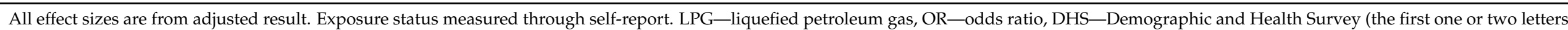
represent country name initials), NFHS-National Family Health Survey.

Table 4. DHS data-based evidence on the association between cooking fuel use and birthweight.

\begin{tabular}{|c|c|c|c|c|c|c|c|c|c|}
\hline $\begin{array}{l}\text { Authors and } \\
\text { Publication } \\
\text { Year }\end{array}$ & $\begin{array}{l}\text { Country and } \\
\text { Survey Year }\end{array}$ & $\begin{array}{l}\text { Unit of } \\
\text { Analyses }\end{array}$ & Sample Size & Exposed Group & $\begin{array}{l}\text { Comparator } \\
\text { Group }\end{array}$ & $\begin{array}{l}\text { Outcome and } \\
\text { Definition }\end{array}$ & $\begin{array}{c}\text { Covariate } \\
\text { Adjusted (Table } 1 \\
\text { Footnote) }\end{array}$ & $\begin{array}{c}\text { Statistical Method } \\
\text { and } \\
\text { Main Results }\end{array}$ & $\begin{array}{c}\text { Risk of } \\
\text { BiasScore }\end{array}$ \\
\hline $\begin{array}{l}\text { Milanzi et al. } \\
\text { (2017) }\end{array}$ & $\begin{array}{l}\text { Malawi, } 2010 \\
\text { MDHS }\end{array}$ & $\begin{array}{l}\text { Under-five } \\
\text { children }\end{array}$ & 9124 & $\begin{array}{c}\text { Highly } \\
\text { polluting fuels: } \\
\text { charcoal, wood, } \\
\text { crop waste, } \\
\text { straw or dung }\end{array}$ & $\begin{array}{l}\text { Low pollution } \\
\text { fuels: electricity, } \\
\text { LPG or biogas }\end{array}$ & $\begin{array}{c}\text { Birthweight } \\
\text { from health card } \\
\text { and size at birth } \\
\text { recalled }\end{array}$ & $\begin{array}{c}2,3,14,15,18,30 \\
52 \text { and } 50\end{array}$ & $\begin{array}{c}\text { Linear plus logistic } \\
\text { regression } \\
\text { OR, } 95 \% \mathrm{CI} \\
\text { birthweight } \\
\text { (continuous): } 92 \mathrm{~g} \\
(-320.4 ; 136.4) \\
\text { Size at birth } \\
\text { (binary): } 1.29(0.34 ; \\
4.80)\end{array}$ & Serious \\
\hline
\end{tabular}


Table 4. Cont.

\begin{tabular}{|c|c|c|c|c|c|c|c|c|c|}
\hline $\begin{array}{c}\text { Authors and } \\
\text { Publication } \\
\text { Year }\end{array}$ & $\begin{array}{l}\text { Country and } \\
\text { Survey Year }\end{array}$ & $\begin{array}{l}\text { Unit of } \\
\text { Analyses }\end{array}$ & Sample Size & Exposed Group & $\begin{array}{l}\text { Comparator } \\
\text { Group }\end{array}$ & $\begin{array}{l}\text { Outcome and } \\
\text { Definition }\end{array}$ & $\begin{array}{c}\text { Covariate } \\
\text { Adjusted (Table } 1 \\
\text { Footnote) }\end{array}$ & $\begin{array}{c}\text { Statistical Method } \\
\text { and } \\
\text { Main Results }\end{array}$ & $\begin{array}{c}\text { Risk of } \\
\text { BiasScore }\end{array}$ \\
\hline $\begin{array}{l}\text { Khan et al. } \\
\text { (2017) }\end{array}$ & $\begin{array}{l}\text { Bangladesh, } \\
\text { 2007-2014 } \\
\text { BDHS }\end{array}$ & $\begin{array}{l}\text { Under-five } \\
\text { children }\end{array}$ & 22,789 & $\begin{array}{l}\text { Solid fuel: coal, } \\
\text { lignite, charcoal, } \\
\text { wood, } \\
\text { straw/shrubs/gras } \\
\text { crop waste, } \\
\text { dung or others }\end{array}$ & $\begin{array}{c}\text { Clean fuels: } \\
\text { electricity, LPG, } \\
\text { s, natural gas or } \\
\text { biogas }\end{array}$ & $\begin{array}{l}\text { Birthweight } \\
\text { from health } \\
\text { card, maternal } \\
\text { recall and size at } \\
\text { birth }\end{array}$ & $\begin{array}{c}2,6,14,15,50,51 \\
\text { and } 52\end{array}$ & $\begin{array}{c}\text { Logistic regression } \\
\text { OR, 95\% CI: } \\
1.33(1.14-1.56)\end{array}$ & Serious \\
\hline $\begin{array}{l}\text { Epstein et al. } \\
\text { (2013) }\end{array}$ & $\begin{array}{c}\text { India, 2005-6 } \\
\text { NFHS }\end{array}$ & $\begin{array}{l}\text { Under-five } \\
\text { children } \\
\text { (singleton } \\
\text { recent birth) }\end{array}$ & 14,850 & $\begin{array}{l}\text { Highly } \\
\text { polluting fuels: } \\
\text { (a) kerosene, } \\
\text { (b) coal/lignite, } \\
\text { (c) biomass fuels } \\
\text { (wood, charcoal, } \\
\text { crop waste, } \\
\text { dung or } \\
\text { straw/shrubs/grass }\end{array}$ & $\begin{array}{l}\text { Low polluting } \\
\text { fuels: LPG, } \\
\text { natural gas or } \\
\text { biogas } \\
\text { s) }\end{array}$ & $\begin{array}{l}\text { Birthweight } \\
\text { from health card }\end{array}$ & $\begin{array}{c}2,14,15,16,23,18 \\
50,51,59,54,63,8 \\
\quad 10 \text { and } 29\end{array}$ & $\begin{array}{c}\text { Logistic regression } \\
\text { OR }(95 \% \text { CI): } \\
\text { Kerosene: } 1.51(1.08, \\
2.12) \\
\text { Biomass: } 1.51(1.08, \\
2.12) \\
\text { Coal: } 1.57(1.03, \\
2.41)\end{array}$ & Moderate \\
\hline $\begin{array}{l}\text { Mishra et al. } \\
\text { (2004) }\end{array}$ & $\begin{array}{c}\text { Zimbabwe, } 1999 \\
\text { ZDHS }\end{array}$ & $\begin{array}{l}\text { Under-five } \\
\text { children } \\
\text { (singleton } \\
\text { births) }\end{array}$ & 3331 & $\begin{array}{c}\text { Highly } \\
\text { polluting fuels: } \\
\text { wood, dung, or } \\
\text { straw, and } \\
\text { Medium } \\
\text { polluting fuels: } \\
\text { kerosene or } \\
\text { charcoal } \\
\text { (presented as a } \\
\text { descriptive } \\
\text { only) }\end{array}$ & $\begin{array}{l}\text { Low polluting } \\
\text { fuels: LPG, } \\
\text { natural gas or } \\
\text { electricity }\end{array}$ & $\begin{array}{l}\text { Birthweight } \\
\text { from health card } \\
\text { or mother's } \\
\text { recall }\end{array}$ & $\begin{array}{c}2,3,14,15,30,18 \\
19,21,51 \text { and } 52\end{array}$ & $\begin{array}{l}\text { Logistic regression } \\
\text { OR }(95 \% \mathrm{CI}) \text { : } \\
\text { from a health card } \\
-120 \mathrm{~g}(-301,61) \\
\text { from recall } \\
-183 \mathrm{~g}(-376,10)\end{array}$ & Serious \\
\hline
\end{tabular}

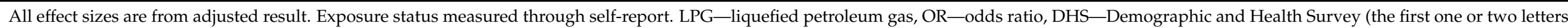
represent country name initials), NFHS-National Family Health Survey. 
Table 5. DHS data-based evidence on the association between cooking fuel use and adverse pregnancy and birth outcomes.

\begin{tabular}{|c|c|c|c|c|c|c|c|c|c|}
\hline $\begin{array}{l}\text { Authors and } \\
\text { Publication } \\
\text { Year }\end{array}$ & $\begin{array}{l}\text { Country and } \\
\text { Survey Year }\end{array}$ & $\begin{array}{l}\text { Unit of } \\
\text { Analysis }\end{array}$ & Sample Size & Exposed Group & $\begin{array}{l}\text { Comparator } \\
\text { Group }\end{array}$ & $\begin{array}{l}\text { Outcome } \\
\text { Definition }\end{array}$ & $\begin{array}{c}\text { Covariate } \\
\text { Adjusted (Table } 1 \\
\text { Footnote) }\end{array}$ & $\begin{array}{c}\text { Statistical Method } \\
\text { and } \\
\text { Main Results }\end{array}$ & $\begin{array}{c}\text { Risk of } \\
\text { BiasScore }\end{array}$ \\
\hline $\begin{array}{l}\text { Nisha et al. } \\
\text { (2018) }\end{array}$ & $\begin{array}{c}\text { Bangladesh } \\
(2004-2014) \\
\text { BDHS }\end{array}$ & $\begin{array}{l}\text { Singleton } \\
\text { pregnancy }\end{array}$ & 27,237 & $\begin{array}{c}\text { Polluting fuels: } \\
\text { kerosene, } \\
\text { coal/lignite, } \\
\text { charcoal, wood, } \\
\text { straw/shrubs/grass, } \\
\text { crop waste, or dung }\end{array}$ & $\begin{array}{c}\text { Clean fuels: } \\
\text { electricity, LPG, } \\
\text { natural gas, and } \\
\text { biogas }\end{array}$ & $\begin{array}{l}\text { Stillbirth: } \\
\text { Foetal death in } \\
\text { pregnancy of at } \\
\text { least } 7 \text { or more } \\
\text { months }\end{array}$ & $\begin{array}{c}3,14,15,18,24,50 \\
52,47 \text { and } 66\end{array}$ & $\begin{array}{c}\text { Logistic regression } \\
\text { OR 95\% CI: } \\
1.25(0.85,1.84)\end{array}$ & Critical \\
\hline $\begin{array}{l}\text { Khan et al. } \\
\text { (2017) }\end{array}$ & $\begin{array}{c}\text { Bangladesh } \\
(2007-2014) \\
\text { BDHS }\end{array}$ & $\begin{array}{c}\text { Ever } \\
\text { married } \\
\text { women } \\
\text { (10-49 year.) }\end{array}$ & 22,789 & $\begin{array}{c}\text { Solid fuel: } \\
\text { coal/lignite, } \\
\text { charcoal, wood, } \\
\text { straw /shrubs/grass, } \\
\text { crop waste, dung or } \\
\text { others }\end{array}$ & $\begin{array}{c}\text { Clean fuel: } \\
\text { electricity, LPG, } \\
\text { natural gas or } \\
\text { biogas }\end{array}$ & $\begin{array}{l}\text { Stillbirth: } \\
\text { Foetal death } \\
\text { lasting seven or } \\
\text { more months }\end{array}$ & $\begin{array}{c}2,6,14,15,50,51 \\
\text { and } 52\end{array}$ & $\begin{array}{c}\text { Logistic regression } \\
\text { OR 95\% CI: } \\
\text { (OR 0.96: } 0.73,1.27)\end{array}$ & Critical \\
\hline $\begin{array}{l}\text { Mishra et al. } \\
\text { (2005) }\end{array}$ & $\begin{array}{c}\text { India, 1998-99 } \\
\text { NFHS }\end{array}$ & $\begin{array}{c}\text { Ever } \\
\text { married } \\
\text { women aged } \\
\text { 40-49 years }\end{array}$ & 19,189 & $\begin{array}{l}\text { 1. highly polluting } \\
\text { group: only } \\
\text { biomass fuels } \\
\text { (wood, dung, or } \\
\text { crop waste } \\
\text { 2. medium } \\
\text { polluting groups: } \\
\text { mix of biomass } \\
\text { fuels and cleaner } \\
\text { fuels (coal/lignite } \\
\text { or charcoal) }\end{array}$ & $\begin{array}{c}\text { Only cleaner } \\
\text { fuels: electricity, } \\
\text { LPG, biogas, or } \\
\text { kerosene }\end{array}$ & $\begin{array}{c}\text { Stillbirth: } \\
\text { delivery of a } \\
\text { dead baby after } \\
\text { the 28th week of } \\
\text { pregnancy }\end{array}$ & $\begin{array}{l}16,20,15,30,57 \\
47,31,45,52,51 \\
\text { and } 44\end{array}$ & $\begin{array}{c}\text { Logistic regression } \\
\text { OR }(95 \% \text { CI): } \\
\text { Highly polluting } \\
\text { group: } 1.44(1.04, \\
1.97) \\
\text { risk of repeated } \\
\text { incidence of } \\
\text { stillbirth RRR (95\% } \\
\text { CI): } \\
\text { Highly polluting } \\
\text { group: } 2.01(1.11, \\
3.62)\end{array}$ & Critical \\
\hline
\end{tabular}


Table 5. Cont.

\begin{tabular}{|c|c|c|c|c|c|c|c|c|c|c|c|c|c|c|}
\hline $\begin{array}{c}\text { Authors and } \\
\text { Publication } \\
\text { Year }\end{array}$ & $\begin{array}{l}\text { Country and } \\
\text { Survey Year }\end{array}$ & $\begin{array}{l}\text { Unit of } \\
\text { Analysis }\end{array}$ & \multicolumn{2}{|c|}{ Sample Size } & \multicolumn{2}{|c|}{ Exposed Group } & \multicolumn{2}{|c|}{$\begin{array}{l}\text { Comparator } \\
\text { Group }\end{array}$} & \multicolumn{2}{|c|}{$\begin{array}{l}\text { Outcome } \\
\text { Definition }\end{array}$} & \multicolumn{2}{|c|}{$\begin{array}{c}\text { Covariate } \\
\text { Adjusted (Table } 1 \\
\text { Footnote) }\end{array}$} & $\begin{array}{c}\text { Statistical Method } \\
\text { and } \\
\text { Main Results }\end{array}$ & $\begin{array}{c}\text { Risk of } \\
\text { BiasScore }\end{array}$ \\
\hline $\begin{array}{l}\text { Agrawal } \\
(2015)\end{array}$ & $\begin{array}{c}\text { India, 2005-2006 } \\
\text { NFHS }\end{array}$ & $\begin{array}{l}\text { Women } \\
\text { aged 15-49 } \\
\text { (who had } \\
\text { live birth in } \\
\text { the previous } \\
5 \text { years) }\end{array}$ & \multicolumn{2}{|c|}{39,657} & $\begin{array}{r}\text { High and me } \\
\text { exposure gr } \\
\text { biomass fuels } \\
\text { as, wood, c } \\
\text { waste, dur } \\
\text { straw / shrubs, } \\
\text { or solid fuels } \\
\text { as coal/lignit } \\
\text { charcoal }\end{array}$ & $\begin{array}{l}\text { dium } \\
\text { oup: } \\
\text { s such } \\
\text { rop } \\
\text { ng, } \\
\text { /grass, } \\
\text { such } \\
\text { te and } \\
\text { l }\end{array}$ & $\begin{array}{r}\text { Low-ex } \\
\text { group } \\
\text { cleane } \\
\text { (kero } \\
\text { LPG/r } \\
\text { gas, bic } \\
\text { electr }\end{array}$ & $\begin{array}{l}\text { osure } \\
\text { only } \\
\text { fuels } \\
\text { ene, } \\
\text { atural } \\
\text { gas, or } \\
\text { city) }\end{array}$ & \multicolumn{2}{|c|}{$\begin{array}{l}\text { Preeclampsia/Ecla } \\
\text { difficulty with } \\
\text { vision during } \\
\text { daylight, and } \\
\text { swelling of the } \\
\text { legs, body, or } \\
\text { face and } \\
\text { convulsions (not } \\
\text { from fever) } \\
\end{array}$} & \multicolumn{2}{|c|}{$\begin{array}{c}44,9,22,18,16,17 \\
76,77,20,14,15 \\
30,52,50 \text { and } 51\end{array}$} & $\begin{array}{c}\text { Logistic regression } \\
\text { OR }(95 \% \text { CI): } \\
2.21(1.26,3.87)\end{array}$ & Serious \\
\hline \multicolumn{15}{|c|}{$\begin{array}{l}\text { All effect sizes are from adjusted result. Exposure status measured through self-report. LPG—liquefied petroleum gas, OR—odds ratio, DHS—Demographic and Health Survey (the first one or two letters } \\
\text { represent country name initials), NFHS—National Family Health Survey. }\end{array}$} \\
\hline $\begin{array}{c}\text { Authors } \\
\text { and } \\
\text { Publication } \\
\text { Year }\end{array}$ & $\begin{array}{l}\text { Country and } \\
\text { Survey Year }\end{array}$ & $\begin{array}{l}\text { Unit of } \\
\text { Analysis }\end{array}$ & $\begin{array}{l}\text { Sample } \\
\text { Size }\end{array}$ & Exp & sed Group & $\underset{\mathrm{Gr}}{\mathrm{Com}_{1}}$ & $\begin{array}{l}\text { arator } \\
\text { up }\end{array}$ & $\begin{array}{l}\mathrm{Ou} \\
\mathrm{De}\end{array}$ & $\begin{array}{l}\text { come } \\
\text { nition }\end{array}$ & $\begin{array}{r}\text { Co } \\
\text { Adjust } \\
\text { Fo }\end{array}$ & $\begin{array}{l}\text { Jariate } \\
\text { ed (Table } 1 \\
\text { tnote) }\end{array}$ & Sta & $\begin{array}{l}\text { istical Method and } \\
\text { Main Results }\end{array}$ & $\begin{array}{c}\text { Risk of } \\
\text { BiasScore }\end{array}$ \\
\hline $\begin{array}{c}\text { Amegah } \\
\text { et al. (2019) }\end{array}$ & $\begin{array}{c}\text { Ghana, } 2014 \\
\text { GDHS }\end{array}$ & $\begin{array}{l}\text { women aged } \\
15-49 \text { years }\end{array}$ & 4751 & $\begin{array}{r}1 \\
\text { 2. } \mathrm{F} \\
\text { 3. } \mathrm{O} \\
\text { (stra) } \\
\text { or }\end{array}$ & $\begin{array}{l}\text { Charcoal, } \\
\text { rewood and } \\
\text { her biomass } \\
\text { /shrubs/grass } \\
\text { rop waste) }\end{array}$ & $\begin{array}{r}\text { Clea } \\
\text { electric } \\
\text { or nat }\end{array}$ & $\begin{array}{l}\text { fuel: } \\
\text { ity, LPG } \\
\text { iral gas }\end{array}$ & $\begin{array}{r}\text { Redu } \\
\text { weigh }\end{array}$ & $\begin{array}{l}\text { ed body } \\
\text { and BMI }\end{array}$ & $\begin{array}{r}50,40 \\
43,5\end{array}$ & $\begin{array}{l}42,30,31 \\
2 \text { and } 23\end{array}$ & $\begin{array}{r}\text { I } \\
\text { Adjus } \\
\text { Charco } \\
\text { Wood } \\
\text { Othe } \\
\\
\text { Charco } \\
\text { Wood } \\
\text { Othe }\end{array}$ & $\begin{array}{l}\text { inear regression } \\
\text { ed } \beta(95 \% \mathrm{CI}) \text { : Weight } \\
\quad(\text { in } \mathrm{kg}) \\
\mathrm{l}:-3.00(-4.41,-1.60) \\
-7.29(-9.00,-5.58) \\
\text { biomass fuel: }-4.10 \\
(-7.15,1.04) \\
\text { BMI }\left(\mathrm{kg} / \mathrm{m}^{2}\right) \\
\mathrm{l}:-0.78(-1.50,-0.06) \\
-2.27(-2.95,-1.59) \\
\text { biomass fuel: }-0.86 \\
(-2.46,0.75)\end{array}$ & Moderate \\
\hline
\end{tabular}


Table 6. Cont

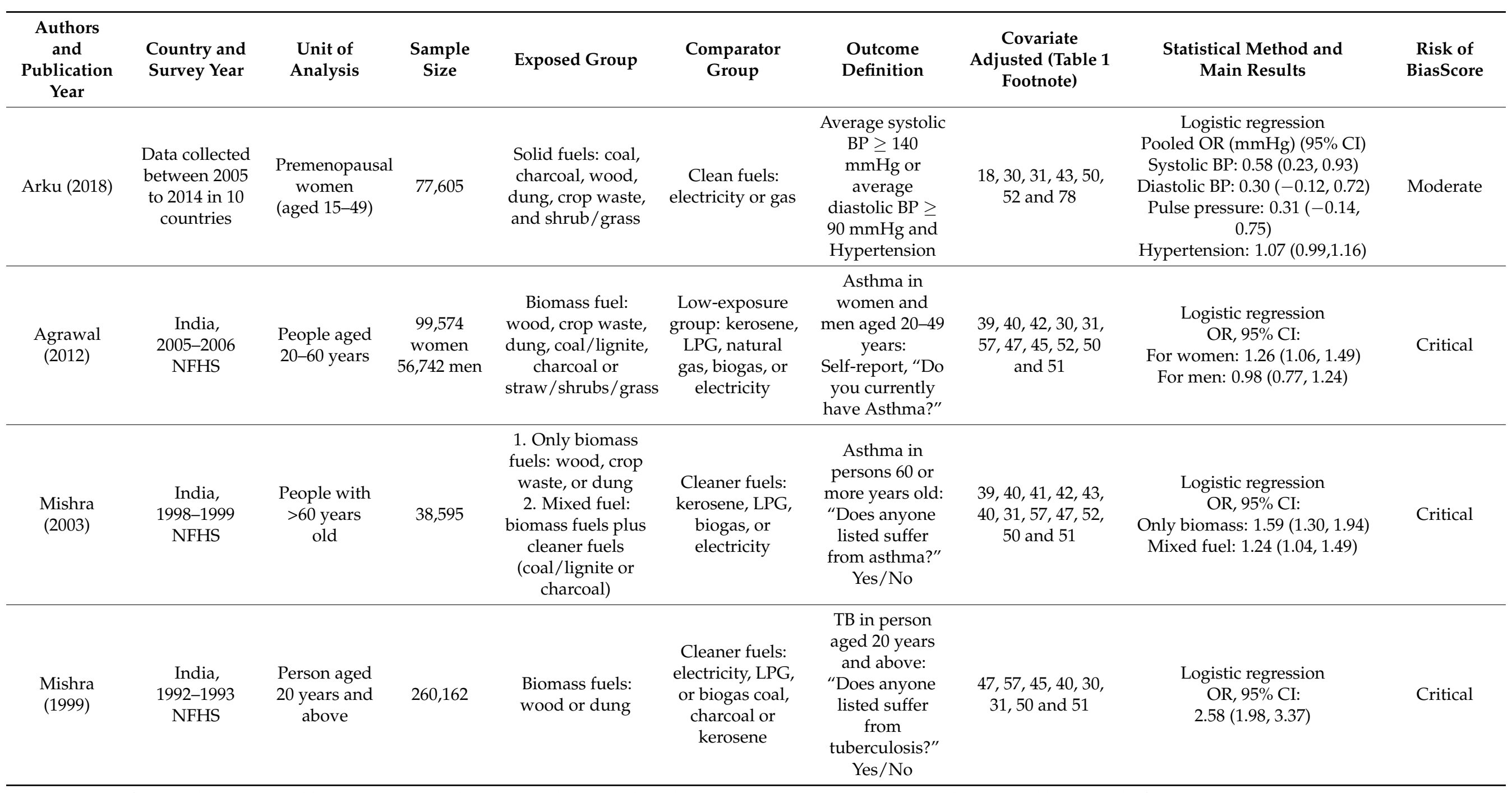

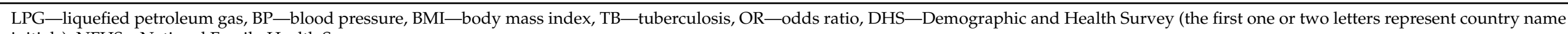
initials), NFHS-National Family Health Survey. 


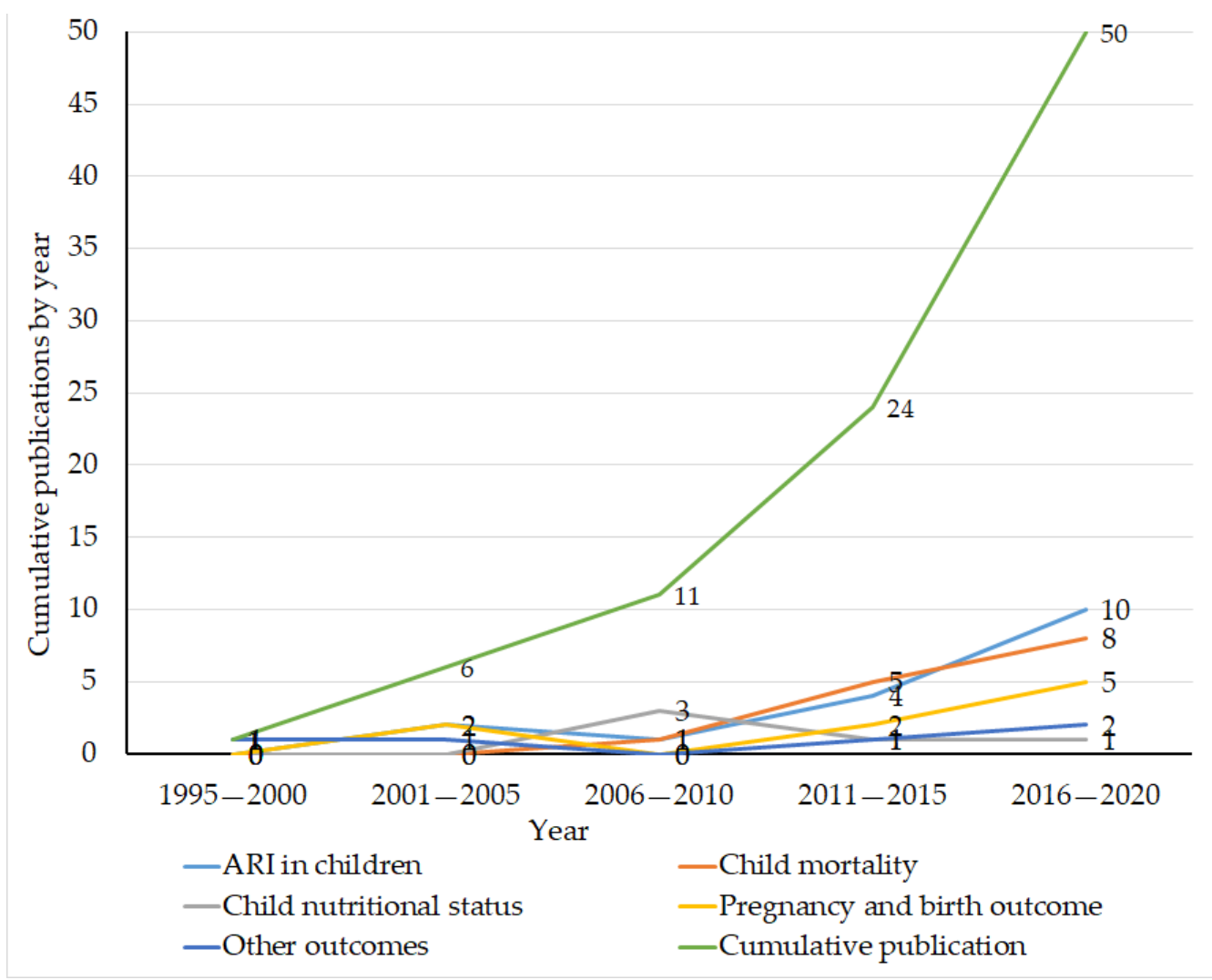

Figure 2. Number of publications reporting effects of household air pollution on health from DHS data, by health outcomes and year of publication, 2020. Note: pregnancy and birth outcomes: birthweight, stillbirth, and preeclampsia/eclampsia. Other health outcomes: asthma (2 studies), maternal body weight (1 study), tuberculosis (1 study) and premenopausal blood pressure (1 study). ARI: acute respiratory infection.

\subsection{Statistical Methods}

As Tables 1-6 highlight, there were inconsistencies in the ways these studies form predictor variable (cooking fuels) — some authors dichotomized it into polluting and clean; others categorised it into different multinomial forms. As a result, there were variations in the definition of exposed and non-exposed (comparator) households across studies. Though there were big dissimilarities across studies in the numbers and types of covariates adjusted, factors related to child, parental, household, health service and environment were observed. All studies of respiratory illnesses utilized the logistic regression approach (Table 1). Of 14 studies of all-cause under-five mortality, 9 used logistic regression, 4 used the Cox regression technique, and 1 used Poisson regression (Table 2). All studies of childhood nutritional status used logistic regression (1 binary and 4 multinomial) (Table 3 ). Linear regression was used by 3 studies of birthweight (Table 4).

\subsection{Under-Five Respiratory Health Studies}

Seventeen out of 50 (34\%) analyses reported the association between cooking fuel use and ARI in children. Based on maternal recall, these studies ascertained ARI using a range of survey-based methods (Table 1). These ascertainment methods included (i) cough accompanied by short and rapid breathing which was coupled with a problem in the chest (7/17 studies), (ii) cough accompanied by short and rapid breathing (the associated problem in the chest not included) (9/17 studies), and (iii) difficulty in breathing and chestrelated congestion and blocked nose and sought-after treatment (1/17 study). However, one study used two of these ascertainment methods (one as a robustness check) [47], all based on one method only. Twelve out of 17 (71\%) studies reported an adverse association, 
while all the remaining studies found no effect. Eight out of 17 (47\%) studies had moderate RoB, of which six reported positive associations. All the studies (three) that had serious RoB, and $3 / 6$ studies that had critical RoB, reported positive associations. All the remaining studies reported no effect (Table 1 ).

\subsection{Studies of All-Cause Under-Five Mortality}

Fourteen out of $50(28 \%)$ analyses reported associations between exposure to smoke from cooking fuels and all-cause child mortality (expressed as odds ratio, relative risk and hazard ratio) (Table 2). Under-five mortality in the DHS is collected based on a synthetic cohort life table approach, which is used to collect the probability of mortality for a small age segments and then combined into the standard age segments [67]. Most of these studies also presented the results of subgroup analysis, based on age, and for neonatal ( $0-28$ days), post-neonatal (1-11 months), child (12-59 months) and under-five (0-59 months). Eleven (78\%) studies reported positive associations between cooking fuel use and death in at least one of these subgroups, while the remaining three studies reported no association. The greatest odds of mortality were observed in neonates (age during the first 28 days of life) that were living in households where cooking was done by polluting fuel, which was reported by Naz and colleagues [38] (OR: 2.67, 95\% CI: 1.47, 4.82), compared to households primarily using low-emission fuels. Additionally, one pooled analysis that collated data from 47 countries [60] found an adverse association between cooking with kerosene (RR: 1.34, 95\% CI: 1.18, 1.52), and solid fuels (RR: 1.24, 95\% CI: 1.14, 1.34) and neonatal death. Based on RoB assessment, $6 / 7$ studies that had moderate RoB reported positive associations. Additionally, $3 / 4$ studies that had serious RoB and $2 / 3$ studies that had critical RoB reported positive associations. All the remaining studies reported no effect (Table 2).

\subsection{Childhood Nutritional Status Studies}

Five out of $50(10 \%)$ analyses assessed the association between cooking fuel use and nutritional status (stunting and anaemia) in children (Table 3). These studies used the recorded age and height of the children to ascertain stunting, and haemoglobin count of the children to ascertain anaemia. These outcome ascertainment methods were robust compared to the methods used to ascertain the other health outcomes included in this review (Table S3). All of these studies categorised kerosene under the low polluting (comparator) group. Three of these studies reported moderate to severe stunting and anaemia relative risk ratios among children living in households where cooking was done with only biomass (wood, crop waste, or dung) $[33,65,66]$, while the remaining two studies reported no effect. Four out of five studies had serious RoB, whilst the remaining one had critical RoB. One of the studies that labelled under serious RoB and a study with critical RoB reported no association (Table 3).

\subsection{Birthweight Studies}

Five out of $50(10 \%)$ analyses described the association of cooking fuel use and birthweight of children (Table 4). These studies used different forms of birthweight data, including weight retrieved from health card (recorded at birth), weight from maternal recall, and child's size at birth (very large, large, average, below average and small), as judged by mothers at the time of interview. One of these studies used birthweight retrieved from the health card only [25], while the remaining studies used more than one of these birthweight data. All birthweight-related studies took households that were using electricity, LPG, or natural gas as a reference (i.e., unexposed to polluting fuels) group. Three out of five studies reported adverse associations, while the remaining two reported no effect. Among these studies, a study which was based on a relatively robust source of outcome information (weight retrieved only from health card) reported that exposure to three polluting fuels was associated with reduced birthweight [25], including kerosene (OR: 1.51, 95\% CI: 1.08, 2.12), biomass (OR: 1.51, 95\% CI: 1.08, 2.12), and coal (OR: 1.57, 95\% 
CI: $1.03,2.41)$. In contrast, another study that used the same source of information on the outcome (conducted separate analysis) [59] found no such association. Overall, four of five studies had serious RoB, and the remaining one had moderate RoB. Of the three studies that reported adverse association, two of them had serious RoB, and the other one had moderate RoB (Table 4).

\subsection{Pregnancy and Birth Complications Related Studies}

Four out of $50(8 \%)$ analyses reported the association between cooking fuel use and pregnancy and birth complications. These studies included stillbirth (three studies) $[23,24,32]$ and preeclampsia/eclampsia (one study) as outcomes [35]. These studies were based on self-report (maternal recall). Two of these studies (one of the stillbirth study and the preeclampsia/eclampsia study) [32,35] reported an adverse association, while the remaining two studies reported no association between solid fuel use and stillbirth. All of the stillbirth-related studies had critical RoB, and the remaining one had serious RoB (Table 5).

\subsection{Other Health Outcomes (Five Studies)}

Table 6 presents the details of studies on other outcomes in addition to those described in the preceding sections. We identified five studies $(10 \%)$, and the outcomes included reduced body weight in adult women [53], elevated blood pressure in pre-menopausal women [64], self-reported asthma among adult men and women (aged 20-49 years) [36], and in persons aged $\geq 60$ years [34] and tuberculosis in adults [30]. Two studies were rated as having moderate RoB, while the rest were rated as having critical RoB. All of the five studies reported positive associations (Table 6).

\section{Discussion}

The Demographic and Health Survey (DHS) is the largest and longest-running source of data on exposure to household air pollution (HAP) in low- and middle-income countries (LMICs), where the availability of vital registration statistics and other administrative records are often scarce. The DHS has a number of attractive features, including national coverage, high quality interviewer training, strong fieldwork coordination, standardised procedures across countries, and high response rates [11,67]. The use of uniform survey instruments allows for the comparison of health and health-related information both within and between countries. In most countries in which data are collected, surveys are repeated periodically, allowing methods and scope to be improved as new data needs and priorities emerge $[11,68]$.

In environmental health research, the survey has seen strongest uptake in studies on water, sanitation and hygiene (WASH) [69] and HAP. Moreover, since 2000, DHS surveys have also collected spatial information on anonymised sampling locations and have been linked to environmental and sociodemographic datasets, both within data supplied by the DHS and by end-users [70,71]. Given the importance of location on exposure to environmental hazards, which can be spatially heterogeneous over small areas, this information has substantial potential to continue to enable a wider variety of studies, and different analytical methods. For studies of HAP from cooking, this can allow researchers to control for potential relevant spatial covariates in their analyses (e.g., air pollutants, temperature, humidity and rainfall, to name a few).

In this review, 45 studies that investigated the cross-sectional associations between cooking fuel use and 11 different health outcomes from 57 countries (there were studies that included more than one country) were included. In total, 36 (80\%) studies reported statistically significant adverse associations between using polluting fuels and health outcomes.

\subsection{Limitations of DHS Surveys (with Respect to HAP and Health)}

The DHS surveys collect data mainly from self-reports through face-to-face interviews, which are prone to reporting and recall biases. Specific to the current review, for example, 
the DHS collects cooking fuel, which is widely used as a proxy to estimate HAP, although non-cooking sources (lighting and heating) are not elicited. The survey uses the 11-item classification of cooking fuel: electricity, liquid petroleum gas (LPG), natural gas, biogas, kerosene, coal/lignite, charcoal, wood, straw/shrubs/grass, crop waste, and animal dung. The question is: "what type of fuel does your household mainly use for cooking?" followed by the above list of fuels. This cooking fuel-related question has several limitations. First, it does not allow multiple responses that could help to capture fuel stacking, which is a common practice in LMICs and in rural settings where wood, dung, charcoal, kerosene, LPG and other fuels used in combination [72-74]. Second, despite the fact that households use polluting fuels, including coal, biomass and kerosene for heating and lighting [75,76], this information was not collected by the DHS surveys. Third, different stoves using the same fuel can vary markedly in their emission characteristics [1], but this level of detail is not collected in the DHS. Cooking emissions also affect outdoor air quality, which is beyond the DHS' remit, but presents an important challenge for researchers attempting to select covariates in their analyses of cooking fuel and health associations. These factors may underestimate the level of exposure and the resultant disease burden in those regions. Moreover, many authors in the included studies mentioned that the survey they lacked some important variables, as measured by indices, such as completeness, detail and absence. For example, a study that pooled data from countries in sub-Saharan Africa indicated a large number of missing observations on cooking fuel $(50.3 \%)$, on cooking location (53.4\%) and on stove ventilation (59.1\%) [63]. The DHS surveys are repeated at approximately five-yearly intervals, but the survey protocol and aims do not seek to target the same households for follow-up in each successive repeat, and longitudinal analyses are, therefore, not possible. Health researchers have performed numerous cross-sectional secondary analyses, but this is an important limitation of the survey in the context of epidemiological studies.

In terms of the health outcomes identified in this review, validation studies have shown that the tool used to ascertain ARI in children has limitations in discerning episodes of the real ARI from simple cough/cold accurately [77,78]. In addition, the studies we reviewed defined ARI in children differently. Since many births in the DHS regions are conducted at home, the weight at birth was missing for a substantial number of birth in the dataset [63]. As a result, three out of five studies included in our review combined three sources of birthweight information in their analyses: birthweight retrieved from birth cards, birthweight recalled by mothers, and child's size at birth (as reported by mothers using an ordinal scale). Whether this is more valid than using one source of information is unknown, and highlights the broader challenges faced by researchers and the often-ad hoc decisions that make direct comparison between studies difficult, even when using the same data. We reviewed 14 studies of child mortality (neonatal, post-neonatal, infant, child and under-five) and three studies on risk of stillbirth, which depend on accurate age and date of birth and death records but could be affected by age aggregation and inconsistencies in reporting $[79,80]$. Although anthropometric information in the DHS surveys is collected objectively with standardized methods, exposure misclassification (mixing relatively clean and relatively polluting fuels in assigning households to exposed and reference groups) was the main reason studies of childhood stunting and anaemia were rated as having serious RoB.

In general, inadequate control for potentially important covariates, such as ambient air pollutants (e.g., particulate matter, nitrogen oxides, ozone and carbon monoxide), was widely observed across studies. Most of these factors have been identified as important, either independently or as a confounder for respiratory health, mortality, nutritional status, and adverse pregnancy and birth outcomes. This gap might happen because of the fact that data on these ambient air pollutants do not exist in the existing DHS dataset, but can be linked from other sources using spatial information for sampling locations. Moreover, the covariates that are collected in many DHS surveys (e.g., environmental temperature and humidity) and known confounders for some of the child health studies (e.g., ARI and 
nutrition) $[81,82]$ were not adequately considered in most studies. These variables are available in the majority of DHS surveys that were collected after 2000. Only two studies had attempted to control seasonal variations by taking the data collection/interview period as a season in a year in their analyses $[45,63]$. As a result, the majority of included studies were found to have moderate to critical RoB for the confounding domain in the itemlevel RoB assessment. Taking the confounding effect of these variables (at least those that are collected in the DHS surveys) into account could help to reduce such RoB. The misclassification of exposure status was also widely observed. More than one-third of the included studies classified kerosene under clean (low emission) fuels. However, the $\mathrm{WHO}$, in its indoor air quality guidelines, regarded kerosene as a polluting fuel in 2014 [1]. One-third of those studies that considered kerosene as a low emission fuel were published after these guidelines. In terms of outcome ascertainment methods, with the exception of height and weight (used to determine nutritional status) and haemoglobin count (used to determine anaemia), all measures in the DHS are captured from maternal/caregivers recall (e.g., ARI and mortality in children) and self-reporting (e.g., pregnancy and birth complications). As a result, bias related to the misclassification of the outcome could be significant. In addition, studies included in this review used different definitions for the same outcome (e.g., ARI in children and weight at birth). This risk of bias could be minimised by using any available guideline; for example, the revised version of pneumonia guidelines for ARI in children [83].

\subsection{Strengths and Limitations of this Review}

We reviewed studies based on the DHS because it is an important, often sole, source of data on HAP and health outcomes in LMICs. We used a new risk of bias assessment tool, which is appropriate for environmental exposure studies, and assessed risk of bias both at study level and at outcome level [22]. In addition to the standard bibliographic databases, we searched evidence under the DHS publication portal. About $90 \%$ of the studies in our review were new (not included in previous reviews) and our review entirely focused on epidemiological studies obtained from further analyses of DHS data. We hope our findings and suggestions are helpful to end users of DHS data, as well as the large global team tasked with designing and administering the crucial work of the DHS program. To that end, we have summarised our impression of the main challenges, together with some suggestions for how they might be addressed, in Table 7.

Table 7. Current challenges and suggested future directions for DHS data used in HAP and health studies.

\begin{tabular}{|c|c|c|c|}
\hline Challenges & $\begin{array}{l}\text { Suggestions (Survey Planning and } \\
\text { Implementation/Policy/Research) }\end{array}$ & Priority to Implement & Difficulty to Implement \\
\hline $\begin{array}{l}\text { Data on the degree of } \\
\text { exposure to smoke from } \\
\text { cooking fuels collected using } \\
\text { questionnaire, which is likely } \\
\text { to be less accurate than other } \\
\text { methods of exposure } \\
\text { assessment. }\end{array}$ & $\begin{array}{l}\text { DHS and other household surveys } \\
\text { could implement more robust } \\
\text { methods (e.g., biological monitoring, } \\
\text { personal sampling and } \\
\text { micro-environmental area-based } \\
\text { sampling) in a smaller, representative } \\
\text { sub-sample. }\end{array}$ & High & Medium \\
\hline $\begin{array}{l}\text { DHS collects a single (main) } \\
\text { fuel item used for cooking, } \\
\text { but households use multiple } \\
\text { fuel items (fuel stacking) for } \\
\text { cooking, and for other } \\
\text { purposes (e.g., heating and } \\
\text { lighting). }\end{array}$ & $\begin{array}{l}\text { Modifying the DHS questionnaire in } \\
\text { a way that it can capture multiple } \\
\text { responses on the types of fuel used } \\
\text { for cooking and for other purposes } \\
\text { would be beneficial, including more } \\
\text { granular estimates of exposure to } \\
\text { cooking-derived pollutant (either } \\
\text { categorical or continuous, from } \\
\text { models and/or measurements). }\end{array}$ & High & Low \\
\hline
\end{tabular}


Table 7. Cont.

\begin{tabular}{|c|c|c|c|}
\hline Challenges & $\begin{array}{l}\text { Suggestions (Survey Planning and } \\
\text { Implementation/Policy/Research) }\end{array}$ & Priority to Implement & Difficulty to Implement \\
\hline $\begin{array}{l}\text { Included studies did not } \\
\text { control for some potentially } \\
\text { important ambient air } \\
\text { pollutants (e.g., particulate } \\
\left.\text { matter, } \mathrm{NO}_{2}, \mathrm{O}_{3} \text { and } \mathrm{CO}\right) \text {. }\end{array}$ & $\begin{array}{l}\text { Existing public domain, global spatial } \\
\text { exposure datasets (e.g., those used in } \\
\text { the GBD and other studies) could be } \\
\text { linked to geocoded DHS data, which } \\
\text { would reduce the need for individual } \\
\text { researchers to seek out the data and } \\
\text { complete this linkage themselves. }\end{array}$ & Medium & Low \\
\hline $\begin{array}{l}\text { Most of the outcomes, except } \\
\text { child nutritional status and a } \\
\text { few birth weight outcomes, } \\
\text { were based on self-report and } \\
\text { prone to recall bias or have } \\
\text { unclear validity. }\end{array}$ & $\begin{array}{l}\text { Though it is very challenging, and } \\
\text { unlikely to be achieved in the near } \\
\text { future, a shift to collecting more } \\
\text { objectively determined outcomes, } \\
\text { either measured directly or through } \\
\text { linkage with non-DHS administrative } \\
\text { data, would enable a larger range and } \\
\text { higher quality of analyses. }\end{array}$ & High & High \\
\hline $\begin{array}{l}\text { The included studies } \\
\text { collectively suggest that } \\
\text { missing data on cooking fuel } \\
\text { and associated information } \\
\text { (e.g., location of the kitchen } \\
\text { and stove ventilation) can be } \\
\text { make analyses problematic. }\end{array}$ & $\begin{array}{l}\text { Explore implications of missing } \\
\text { exposure data on health analyses and } \\
\text { compare the utility of different } \\
\text { imputation or prediction-based } \\
\text { methods to deal with missingness. }\end{array}$ & High & Low \\
\hline
\end{tabular}

This review has several limitations. One of the limitations of our review is that we have included peer-reviewed research articles published only in the English language. As the DHS surveys are also conducted in non-English speaking countries and the survey information is widely used to develop government documents (e.g., working papers), we might have missed grey literature and some records published in languages other than English. However, we do not think this would alter our substantive findings, as the data collected under the DHS program, and used by researchers, are similar. Finally, for practical reasons, the RoB assessment was undertaken by one of us, and despite using a standard process for assessing each article's RoB, errors or omissions may mean the RoB has been under- or over-estimated for individual studies.

\section{Conclusions}

Although the HAP exposure assessment is not the main focus of the DHS, it is the main, often only, source of information in many low- and middle-income countries. It remains an important resource, and researchers have used it to perform important crosssectional analyses on HAP from cooking fuels and health in 57 countries. By restricting our focus to DHS-based studies, our review allowed for greater granularity in our assessment of DHS-specific issues. Through that, we found that an appreciable proportion of the studies we reviewed have potential for serious or critical RoB, due mainly to the inherent limitations of what information can feasibly be collected in such a large-scale survey, and methodological choices by end-users. This relates to confounder control, exposure misclassification, and outcome ascertainment. We underscore to researchers' need to bear these issues in mind in their analyses. Moreover, we also offer some pragmatic suggestions through which DHS data could be even more amenable to studies of household fuel use and health, and reduce the RoB, without being onerous to collect or analyse. We hope our review is useful for researchers and others working with DHS data.

Supplementary Materials: The following are available online at https://www.mdpi.com/1660 $-4601 / 18 / 4 / 1411 /$ s1, Table S1: Search terms and strategies, Table S2: Quality and Risk of Bias 
assessment, Table S3: Quality and Risk of Bias results heat map, Table S4: Confounding controlled with their code in the document. The review protocol is available on PROSPERO, registration number: CRD42019137937.

Author Contributions: Conceptualization: D.B.O. and L.D.K.; methodology: D.B.O. and L.D.K.; data analysis: D.B.O.; first draft preparation: D.B.O.; revision and editing: L.D.K. and I.A.Y.; supervision: L.D.K. and I.A.Y.; All authors have read and agreed to the published version of the manuscript.

Funding: This review received no external funding.

Institutional Review Board Statement: Not applicable.

Informed Consent Statement: Not applicable.

Acknowledgments: D.B.O. is supported by The University of Queensland (UQ) (Ph.D. scholarship). The authors thank Scott Macintyre, Public Health Liaison Librarian at UQ, for his support regarding search terms and EndNote data management.

Conflicts of Interest: The authors declare no conflict of interest.

\section{References}

1. WHO. WHO Guidelines for Indoor Air Quality: Household Fuel Combustion; World Health Organization: Geneva, Switzerland, 2014.

2. Health Effects Institute. State of Global Air 2019; Special Report; Health Effects Institute: Boston, MA, USA, 2019.

3. Hanif, I. Energy consumption habits and human health nexus in Sub-Saharan Africa. Environ. Sci. Pollut. Res. Int. 2018, 25, 21701-21712. [CrossRef]

4. James, B.S.; Shetty, R.S.; Kamath, A.; Shetty, A. Household cooking fuel use and its health effects among rural women in southern India-A cross-sectional study. PLoS ONE 2020, 15, e0231757. [CrossRef] [PubMed]

5. Adaji, E.E.; Ekezie, W.; Clifford, M.; Phalkey, R. Understanding the effect of indoor air pollution on pneumonia in children under 5 in low- and middle-income countries: A systematic review of evidence. Environ. Sci. Pollut. Res. Int. 2019, 26, $3208-3225$. [CrossRef] [PubMed]

6. Murray, C.J.L.; Aravkin, A.Y.; Zheng, P.; Abbafati, C.; Abbas, K.M.; Abbasi-Kangevari, M.; Abd-Allah, F.; Abdelalim, A.; Abdollahi, M.; Abdollahpour, I.; et al. Global burden of 87 risk factors in 204 countries and territories, 1990-2019: A systematic analysis for the Global Burden of Disease Study 2019. Lancet 2020, 396, 1223-1249. [CrossRef]

7. Balakrishnan, K.; Dey, S.; Gupta, T.; Dhaliwal, R.S.; Brauer, M.; Cohen, A.J.; Stanaway, J.D.; Beig, G.; Joshi, T.K.; Aggarwal, A.N.; et al. The impact of air pollution on deaths, disease burden, and life expectancy across the states of India: The Global Burden of Disease Study 2017. Lancet Planet. Health 2019, 3, e26-e39. [CrossRef]

8. Okello, S.; Akello, S.J.; Dwomoh, E.; Byaruhanga, E.; Opio, C.K.; Zhang, R.; Corey, K.E.; Muyindike, W.R.; Ocama, P.; Christiani, D.D. Biomass fuel as a risk factor for esophageal squamous cell carcinoma: A systematic review and meta-analysis. Environ. Health 2019, 18, 60. [CrossRef]

9. Williams, K.N.; Northcross, A.L.; Graham, J.P. Health impacts of household energy use: Indicators of exposure to air pollution and other risks. Bull. World Health Organ. 2015, 93, 507-508. [CrossRef] [PubMed]

10. ICF. The Sustainable Development Goals. The DHS Program website. Funded by USAID. Available online: http://www. dhsprogram.com (accessed on 19 November 2019).

11. Arnold, F.; Khan, S.M. Perspectives and implications of the Improving Coverage Measurement Core Group's validation studies for household surveys. J. Glob. Health 2018, 8, 010606. [CrossRef]

12. Short Fabic, M.; Choi, Y.; Bird, S. A systematic review of Demographic and Health Surveys: Data availability and utilization for research. Bull. World Health Organ. 2012, 90, 604-612. [CrossRef]

13. Stanaway, J.D.; Afshin, A.; Gakidou, E.; Lim, S.S.; Abate, D.; Abate, K.H.; Abbafati, C.; Abbasi, N.; Abbastabar, H.; Abd-Allah F.; et al. Global, regional, and national comparative risk assessment of 84 behavioural, environmental and occupational, and metabolic risks or clusters of risks for 195 countries and territories, 1990-2017: A systematic analysis for the Global Burden of Disease Study 2017. Lancet 2018, 392, 1923-1994.

14. Smith, K.R.; Bruce, N.; Balakrishnan, K.; Adair-Rohani, H.; Balmes, J.; Chafe, Z.; Dherani, M.; Hosgood, H.D.; Mehta, S.; Pope, D.; et al. Millions dead: How do we know and what does it mean? Methods used in the comparative risk assessment of household air pollution. Annu. Rev. Public Health 2014, 35, 185-206. [CrossRef] [PubMed]

15. Emmelin, A.; Wall, S. Indoor air pollution: A poverty-related cause of mortality among the children of the world. Chest 2007, 132, 1615-1623. [CrossRef] [PubMed]

16. Lin, H.H.; Ezzati, M.; Murray, M. Tobacco smoke, indoor air pollution and tuberculosis: A systematic review and meta-analysis. PLoS Med. 2007, 4, e20. [CrossRef] [PubMed]

17. Pope, D.P.; Mishra, V.; Thompson, L.; Siddiqui, A.R.; Rehfuess, E.A.; Weber, M.; Bruce, N.G. Risk of low birth weight and stillbirth associated with indoor air pollution from solid fuel use in developing countries. Epidemiol. Rev. 2010, 32, 70-81. [CrossRef] [PubMed] 
18. Po, J.Y.; FitzGerald, J.M.; Carlsten, C. Respiratory disease associated with solid biomass fuel exposure in rural women and children: Systematic review and meta-analysis. Thorax 2011, 66, 232-239. [CrossRef] [PubMed]

19. Bruce, N.G.; Dherani, M.K.; Das, J.K.; Balakrishnan, K.; Adair-Rohani, H.; Bhutta, Z.A.; Pope, D. Control of household air pollution for child survival: Estimates for intervention impacts. BMC Public Health 2013, 13 (Suppl 3), S8. [CrossRef]

20. Liberati, A.; Altman, D.G.; Tetzlaff, J.; Mulrow, C.; Gotzsche, P.C.; Ioannidis, J.P.; Clarke, M.; Devereaux, P.J.; Kleijnen, J.; Moher, D. The PRISMA statement for reporting systematic reviews and meta-analyses of studies that evaluate health care interventions: Explanation and elaboration. PLoS Med. 2009, 6, e1000100. [CrossRef]

21. Household Air Pollution and Health Outcomes: A Systematic Review of Global Epidemiological Evidence from the Demographic and Health Surveys (DHS). Available online: https://www.crd.york.ac.uk/prospero/\#recordDetails (accessed on 7 August 2019).

22. Morgan, R.L.; Thayer, K.A.; Santesso, N.; Holloway, A.C.; Blain, R.; Eftim, S.E.; Goldstone, A.E.; Ross, P.; Ansari, M.; Akl, E.A.; et al. A risk of bias instrument for non-randomized studies of exposures: A users' guide to its application in the context of GRADE. Environ. Int. 2019, 122, 168-184. [CrossRef]

23. Khan, M.N.; Nurs, C.Z.B.; Islam, M.M.; Islam, M.R.; Rahman, M.M. Household air pollution from cooking and risk of adverse health and birth outcomes in Bangladesh: A nationwide population-based study. Environ. Health Global Access Sci. Sour. 2017, 16, 57. [CrossRef]

24. Nisha, M.K.; Alam, A.; Raynes-Greenow, C. Variations in perinatal mortality associated with different polluting fuel types and kitchen location in Bangladesh. Int. J. Occup. Environ. Health 2018, 24, 47-54. [CrossRef]

25. Epstein, M.B.; Bates, M.N.; Arora, N.K.; Balakrishnan, K.; Jack, D.W.; Smith, K.R. Household fuels, low birth weight, and neonatal death in India: The separate impacts of biomass, kerosene, and coal. Int. J. Hyg. Environ. Health 2013, 216, 523-532. [CrossRef] [PubMed]

26. Sreeramareddy, C.T.; Shidhaye, R.R.; Sathiakumar, N. Association between biomass fuel use and maternal report of child size at birth-an analysis of 2005-06 India Demographic Health Survey data. BMC Public Health 2011, 11, 10. [CrossRef] [PubMed]

27. Patel, A.B.; Dhande, L.A.; Pusdekar, Y.V.; Borkar, J.A.; Badhoniya, N.B.; Hibberd, P.L. Childhood illness in households using biomass fuels in India: Secondary data analysis of nationally representative national family health surveys. Int. J. Occup. Environ. Health 2013, 19, 35-42. [CrossRef] [PubMed]

28. Pandey, S.; Lin, Y. Adjusted effects of domestic violence, tobacco use, and indoor air pollution from use of solid fuel on child mortality. Matern. Child Health J. 2013, 17, 1499-1507. [CrossRef]

29. Naz, S.; Page, A.; Agho, K.E. Household air pollution and under-five mortality in India (1992-2006). Environ. Health Glob. Access Sci. Sour. 2016, 15, 54. [CrossRef]

30. Mishra, V.K.; Retherford, R.D.; Smith, K.R. Biomass Cooking Fuels and Prevalence of Tuberculosis in India. Int. J. Infect. Dis. 1999, 3, 119-129.

31. Mishra, V.; Smith, K.R.; Retherford, R.D. Effects of cooking smoke and environmental tobacco smoke on acute respiratory infections in young Indian children. Popul. Environ. 2005, 26, 375-396. [CrossRef]

32. Mishra, V.; Retherford, R.D.; Smith, K.R. Cooking smoke and tobacco smoke as risk factors for stillbirth. Int. J. Environ. Health Res. 2005, 15, 397-410. [CrossRef]

33. Mishra, V.; Retherford, R.D. Does biofuel smoke contribute to anaemia and stunting in early childhood? Int. J. Epidemiol. 2007, 36, 117-129. [CrossRef]

34. Mishra, V. Effect of indoor air pollution from biomass combustion on prevalence of asthma in the elderly. Environ. Health Perspect. 2003, 111, 71-78. [CrossRef]

35. Agrawal, S.; Yamamoto, S. Effect of Indoor air pollution from biomass and solid fuel combustion on symptoms of preeclampsia/eclampsia in Indian women. Indoor Air 2015, 25, 341-352. [CrossRef] [PubMed]

36. Agrawal, S. Effect of indoor air pollution from biomass and solid fuel combustion on prevalence of self-reported asthma among adult men and women in India: Findings from a nationwide large-scale cross-sectional survey. J. Asthma 2012, 49, 355-365. [CrossRef]

37. Mondal, D.; Paul, P. Effects of indoor pollution on acute respiratory infections among under-five children in India: Evidence from a nationally representative population-based study. PLOS ONE 2020, 15, e0237611. [CrossRef] [PubMed]

38. Naz, S.; Page, A.; Agho, K.E. Potential Impacts of Modifiable Behavioral and Environmental Exposures on Reducing Burden of Under-five Mortality Associated with Household Air Pollution in Nepal. Matern. Child Health J. 2018, 22, 59-70. [CrossRef] [PubMed]

39. Dadras, O.; Chapman, R.S. Biomass fuel smoke and stunting in early childhood: Finding from a national survey Nepal. J. Health Res. 2017, 31, S7.

40. Acharya, P.; Mishra, S.R.; Berg-Beckhoff, G. solid fuel in kitchen and acute respiratory tract infection among under five children: evidence from Nepal Demographic and Health Survey 2011. J. Community Health 2015, 40, 515-521. [CrossRef]

41. Budhathoki, S.S.; Tinkari, B.S.; Bhandari, A.; Dhimal, M.; Zhou, H.; Ghimire, A.; Basnet, O.; Wrammert, J.; Kc, A. The Association of Childhood Pneumonia with Household Air Pollution in Nepal: Evidence from Nepal Demographic Health Surveys. Matern Child. Health J. 2020, 24 (Suppl. 1), 48-56. [CrossRef]

42. Naz, S.; Page, A.; Agho, K.E. Household air pollution and under-five mortality in Bangladesh (2004-2011). Int. J. Environ. Res. Public Health 2015, 12, 12847-12862. [CrossRef] 
43. Samuel, O.W.; Oni, G.A.; KC, S.; Wurzer, M.; Akinyemi, A.I. Household use of solid fuel for cooking and under-five mortality in Nigeria. Afr. Popul. Stud. 2018, 32, 4034-4042.

44. Ezeh, O.K.; Agho, K.E.; Dibley, M.J.; Hall, J.J.; Page, A.N. The effect of solid fuel use on childhood mortality in Nigeria: Evidence from the 2013 cross-sectional household survey. Environ. Health Global Access Sci. Sour. 2014, 13, 113. [CrossRef]

45. Akinyemi, J.O.; Morakinyo, O.M. Household environment and symptoms of childhood acute respiratory tract infections in Nigeria, 2003-2013: A decade of progress and stagnation. BMC Infect. Dis. 2018, 18, 12. [CrossRef] [PubMed]

46. Naz, S.; Page, A.; Agho, K.E. Household air pollution from use of cooking fuel and under-five mortality: The role of breastfeeding status and kitchen location in Pakistan. PLoS ONE 2017, 12, e0173256. [CrossRef] [PubMed]

47. Khan, M.S.B.; Lohano, H.D. Household air pollution from cooking fuel and respiratory health risks for children in Pakistan. Environ. Sci. Pollut. Res. 2018, 25, 24778-24786. [CrossRef] [PubMed]

48. Naz, L.; Ghimire, U. Assessing the prevalence trend of childhood pneumonia associated with indoor air pollution in Pakistan. Environ. Sci. Pollut. Res. Int. 2020, 27, 44540-44541. [CrossRef]

49. Wichmann, J.; Voyi, K.V.V. Impact of cooking and heating fuel use on acute respiratory health of preschool children in South Africa. South. Afr. J. Epidemiol. Infect. 2015, 21, 48-54. [CrossRef]

50. Wichmann, J.; Voyi, K.V. Influence of cooking and heating fuel use on 1-59 month old mortality in South Africa. Matern. Child Health J. 2006, 10, 553-561. [CrossRef]

51. Mishra, V.; Dai, X.L.; Smith, K.R.; Mika, L. Maternal exposure to biomass smoke and reduced birth weight in Zimbabwe. Ann. Epidemiol. 2004, 14, 740-747. [CrossRef]

52. Mishra, V. Indoor air pollution from biomass combustion and acute respiratory illness in preschool age children in Zimbabwe. Int. J. Epidemiol. 2003, 32, 847-853. [CrossRef]

53. Amegah, A.K.; Boachie, J.; Näyhä, S.; Jaakkola, J.J.K. Association of biomass fuel use with reduced body weight of adult Ghanaian women. J. Expos. Sci. Environ. Epidemiol. 2019, 30, 670-679. [CrossRef]

54. Capuno, J.J.; Tan, C.A.R., Jr.; Javier, X. Cooking and coughing: Estimating the effects of clean fuel for cooking on the respiratory health of children in the Philippines. Global Public Health 2018, 13, 20-34. [CrossRef]

55. Kilabuko, J.H.; Nakai, S. Effects of Cooking Fuels on Acute Respiratory Infections in Children in Tanzania. Int. J. Environ. Res. Public Health 2007, 4, 283-288. [CrossRef] [PubMed]

56. Machisa, M.; Wichmann, J.; Nyasulu, P.S. Biomass fuel use for household cooking in Swaziland: Is there an association with anaemia and stunting in children aged 6-36 months? Trans. R. Soc. Trop. Med. Hyg. 2013, 107, 535-544. [CrossRef] [PubMed]

57. Woolley, K.E.; Bagambe, T.; Singh, A.; Avis, W.R.; Kabera, T.; Weldetinsae, A.; Mariga, S.T.; Kirenga, B.; Pope, F.D.; Thomas, G.N.; et al. Investigating the association between wood and charcoal domestic cooking, respiratory symptoms and acute respiratory infections among children aged under 5 years in Uganda: A cross-sectional analysis of the 2016 demographic and health survey. Int. J. Environ. Res. Public Health 2020, 17, 3974. [CrossRef] [PubMed]

58. Rana, J.; Uddin, J.; Peltier, R.; Oulhote, Y. Associations between indoor air pollution and acute respiratory infections among under-five children in afghanistan: do ses and sex matter? Int J. Environ. Res. Public Health 2019, 16, 2910. [CrossRef]

59. Milanzi, E.B.; Namacha, N.M. Maternal biomass smoke exposure and birth weight in Malawi: Analysis of data from the 2010 Malawi Demographic and Health Survey. Malawi Med. J. 2017, 29, 160-165. [CrossRef]

60. Kleimola, L.B.; Patel, A.B.; Borkar, J.A.; Hibberd, P.L. Consequences of household air pollution on child survival: Evidence from demographic and health surveys in 47 countries. Int. J. Occup. Environ. Health 2015, 21, 294-302. [CrossRef]

61. Owili, P.O.; Muga, M.A.; Pan, W.C.; Kuo, H.W. Cooking fuel and risk of under-five mortality in 23 Sub-Saharan African countries: A population-based study. Int. J. Environ. Health Res. 2017, 27, 191-204. [CrossRef]

62. Akinyemi, J.O.; Adedini, S.A.; Wandera, S.O.; Odimegwu, C.O. Independent and combined effects of maternal smoking and solid fuel on infant and child mortality in sub-Saharan Africa. Trop. Med. Int. Health 2016, 21, 1572-1582. [CrossRef]

63. Buchner, H.; Rehfuess, E.A. Cooking and season as risk factors for acute lower respiratory infections in African children: A cross-sectional multi-country analysis. PLoS ONE 2015, 10, e0128933. [CrossRef]

64. Arku, R.E.; Ezzati, M.; Baumgartner, J.; Fink, G.; Zhou, B.; Hystad, P.; Brauer, M. Elevated blood pressure and household solid fuel use in premenopausal women: Analysis of 12 Demographic and Health Surveys (DHS) from 10 countries. Environ. Res. 2018, 160, 499-505. [CrossRef]

65. Kyu, H.H.; Georgiades, K.; Boyle, M.H. Biofuel smoke and child anemia in 29 developing countries: A multilevel analysis. Ann. Epidemiol. 2010, 20, 811-817. [CrossRef] [PubMed]

66. Kyu, H.H.; Georgiades, K.; Boyle, M.H. Maternal smoking, biofuel smoke exposure and child height-for-age in seven developing countries. Int. J. Epidemiol. 2009, 38, 1342-1350. [CrossRef] [PubMed]

67. Croft, T.N.; Marshall, A.M.J.; Allen, C.K. Guide to DHS Statistics; ICF: Rockville, MD, USA, 2018.

68. del Burgo, M.L.M.; Amaral, T.P. Household Health Surveys in Developing Countries: Challenges for Quantitative Analysis (The Case of Demographic and Health Surveys). J Health Med. Econ. 2015, 2. [CrossRef]

69. Headey, D.; Palloni, G. Water, sanitation, and child health: Evidence from subnational panel data in 59 countries. Demography 2019, 56, 729-752. [CrossRef] [PubMed]

70. Reiner, R.C.; Welgan, C.A.; Casey, D.C.; Troeger, C.E.; Baumann, M.M.; Nguyen, Q.P.; Swartz, S.J.; Blacker, B.F.; Deshpande, A.; Mosser, J.F.; et al. Identifying residual hotspots and mapping lower respiratory infection morbidity and mortality in African children from 2000 to 2017. Nat. Microbiol 2019, 4, 2310-2318. [CrossRef] [PubMed] 
71. Burstein, R.; Henry, N.J.; Collison, M.L.; Marczak, L.B.; Sligar, A.; Watson, S.; Marquez, N.; Abbasalizad-Farhangi, M.; Abbasi, M.; Abd-Allah, F.; et al. Mapping 123 million neonatal, infant and child deaths between 2000 and 2017. Nature 2019, 574, 353-358. [CrossRef] [PubMed]

72. Shupler, M.; Hystad, P.; Gustafson, P.; Rangarajan, S.; Mushtaha, M.; Jayachtria, K.G.; Mony, P.K.; Mohan, D.; Kumar, P.; Pvm, L.; et al. Household, community, sub-national and country-level predictors of primary cooking fuel switching in nine countries from the PURE study. Environ. Res. Lett. 2019, 14, 085006. [CrossRef]

73. Rahut, D.B.; Behera, B.; Ali, A.; Marenya, P. A ladder within a ladder: Understanding the factors influencing a household's domestic use of electricity in four African countries. Energy Econ. 2017, 66, 167-181. [CrossRef]

74. Admasie, A.; Kumie, A.; Worku, A.; Tsehayu, W. Household fine particulate matter (PM 2.5 ) concentrations from cooking fuels: The case in an urban setting, Wolaita Sodo, Ethiopia. Air Qual. Atmos. Health 2019, 12, 12-755. [CrossRef]

75. Lam, N.L.; Smith, K.R.; Gauthier, A.; Bates, M.N. Kerosene: A review of household uses and their hazards in low- and middle-income countries. J. Toxicol. Environ. Health B Crit. Rev. 2012, 15, 396-432. [CrossRef]

76. Lam, N.L.; Chen, Y.; Weyant, C.; Venkataraman, C.; Sadavarte, P.; Johnson, M.A.; Smith, K.R.; Brem, B.T.; Arineitwe, J.; Ellis, J.E.; et al. Household light makes global heat: High black carbon emissions from kerosene wick lamps. Environ. Sci. Technol. 2012, 46, 13531-13538. [CrossRef] [PubMed]

77. Hazir, T.; Begum, K.; El Arifeen, S.; Khan, A.M.; Huque, M.H.; Kazmi, N.; Roy, S.; Abbasi, S.; Rahman, Q.S.; Theodoratou, E.; et al. Measuring coverage in $\mathrm{MNCH}$ : A prospective validation study in Pakistan and Bangladesh on measuring correct treatment of childhood pneumonia. PLoS Med. 2013, 10, e1001422. [CrossRef] [PubMed]

78. Ayede, A.I.; Kirolos, A.; Fowobaje, K.R.; Williams, L.J.; Bakare, A.A.; Oyewole, O.B.; Olorunfemi, O.B.; Kuna, O.; Iwuala, N.T.; Oguntoye, A.; et al. A prospective validation study in South-West Nigeria on caregiver report of childhood pneumonia and antibiotic treatment using Demographic and Health Survey (DHS) and Multiple Indicator Cluster Survey (MICS) questions. J. Glob. Health 2018, 8, 020806. [CrossRef] [PubMed]

79. Lyons-Amos, M.; Stones, T. Trends in Demographic and Health Survey data quality: An analysis of age heaping over time in 34 countries in Sub Saharan Africa between 1987 and 2015. BMC Res. Notes 2017, 10, 760. [CrossRef] [PubMed]

80. Johnson, K.; Grant, M.; Khan, S.; Moore, Z.; Armstrong, A.; Sa, Z. Fieldwork-Related Factors and Data Quality in the Demographic and Health Surveys Program; DHS Analytical Studies No. 19; ICF: Calverton, MD, USA, 2009.

81. Gao, J.; Sun, Y.; Lu, Y.; Li, L. Impact of ambient humidity on child health: A systematic review. PLoS ONE 2014, 9, e112508. [CrossRef]

82. Tusting, L.S.; Bradley, J.; Bhatt, S.; Gibson, H.S.; Weiss, D.J.; Shenton, F.C.; Lindsay, S.W. Environmental temperature and growth faltering in African children: A cross-sectional study. Lancet Planet. Health 2020, 4, e116-e123. [CrossRef]

83. WHO. Revised WHO Classification and Treatment of Childhood Pneumonia at Health Facilities: Evidence Summaries; Maternal, N., Child and Adolescent Health (MCA), Eds.; WHO: Geneva, Switzerland, 2014. 